\title{
Mechanics of finite cracks in dissimilar anisotropic elastic media considering interfacial elasticity
}

\author{
Pierre-Alexandre Juan ${ }^{\mathrm{a}}$, Rémi Dingreville ${ }^{\mathrm{a}, *}$ \\ ${ }^{a}$ Sandia National Laboratories, Albuquerque, NM 87185, USA
}

\begin{abstract}
Interfacial crack fields and singularities in bimaterial interfaces (i.e., grain boundaries or dissimilar materials interfaces) are considered through a general formulation for two-dimensional (2-D) anisotropic elasticity while accounting for the interfacial structure by means of an interfacial elasticity paradigm. The interfacial elasticity formulation introduces boundary conditions that are effectively equivalent to those for a weakly bounded interface. This formalism considers the 2-D crack-tip elastic fields using complex variable techniques. While the consideration of the interfacial elasticity does not affect the order of the singularity, it modifies the oscillatory effects associated with problems involving interface cracks. Constructive or destructive "interferences" are directly affected by the interface structure and its elastic response. This general formulation provides an insight on the physical significance and the obvious coupling between the interface structure and the associated mechanical fields in the vicinity of the crack tip.
\end{abstract}

Keywords: Crack mechanics, Stroh formalism, Anisotropic bimaterial, Interface properties, Surface stress

\section{Introduction}

Solutions to the asymptotic singular fields at an interface crack tip between two materials play a central role in many micromechanics and computational models that are used to study the fracture behavior of engineering materials containing dissimilar material interfaces. Historically, the peculiarity of the near-tip behavior for an interface crack has been studied in both the isotropic bimaterial case (Inglish, 1913; Williams, 1959; Rice, 1988; Shih and Asaro, 1990; Suo and Hutchinson, 1990; Gao,

\footnotetext{
${ }^{*}$ Corresponding author

Email address: rdingre@sandia.gov (Rémi Dingreville)

Preprint submitted to Journal of the Mechanics and Physics of Solids
}

October 7, 2016

(C) 2016. This manuscript version is made available under the Elsevier user license

http://www.elsevier.com/open-access/userlicense/1.0/ 
1991) and the anisotropic case (Gotoh, 1967; Clements, 1971; Willis, 1971; Ting, 1986; Bassani and Qu, 1989a; Qu and Bassani, 1989; Suo, 1990; Wu, 1990; Ni and NematNasser, 1991; Gao et al., 1992) using a variety of mathematical techniques including the Green's function tensor (Gao, 1991), the Stroh formalism (Eshelby et al., 1953; Stroh, 1958, 1962; Deng, 1993), or a distribution of interfacial dislocations (Willis, 1971; Gautesen and Dundurs, 1988; Qu and Li, 1991). Several basic crack problems using these techniques have been solved with an emphasis on the near-tip oscillatory field and the definition of the stress intensity factors for the crack-tip singularity.

In a majority of the referenced works above, the interface is assumed to be perfectly bonded, i.e., those models assume the continuity of both the traction and displacement across the interface. Theoretical models looking into imperfect/weak interfaces have also been developed for both isotropic (Cheng et al., 1996; Antipov et al., 2001; Sudak, 2003) and anisotropic materials (Pan, 2003; Sudak and Wang, 2006; Vellender et al., 2016). These models consider spring-like boundary conditions for which the interfacial displacement jump is directly proportional to the traction. Such boundary conditions at the interface change the character of the crack-tip singularity significantly (Mishuris and Kuhn, 2001).

More recently, due to the growing interests in the so-called field of nanomechanics, the above mentioned seminal developments for perfectly bonded interfaces have been augmented by incorporating surface elasticity effects on the crack surface (Feng et al., 2008; Antipov and Schiavone, 2011; Kim et al., 2011; Nan and Wang, 2012; Koguchi and Suzuki, 2014; Wang et al., 2015; Sigaeva and Schiavone, 2016). The inclusion of the Gurtin and Murdoch (1975) surface elasticity theory in these models has been shown to also modify the nature and functional form of the singularity at the cracktip.

The questions associated with the nature of the fields near a crack-tip are of importance in the context of the Irwin fracture criterion (Irwin, 1957) and require a precise knowledge of the local conditions at the crack-tip. Thus, all of the studies mentioned above (with or without surface elasticity effects) have shown that, for perfectly bonded interfaces, the order of the singularity of a crack lying on the interface is affected by the elastic constants mismatch (c.f. Dundurs (1969) parameters), while the oscillatory singularities are no more than a consequence of the linear elasticity theory. A number of approaches have been proposed to cure this illbehaved elasticity solution. For special combinations of the elastic constants, such as two incompressible materials or one incompressible and the other rigid under plane strain (Comninou, 1990) or for specific mathematical assumptions (England, 1965; Comninou, 1977) on the boundary conditions (frictionless contact near the tip), the elastic fields near the crack-tip follow a square-root singularity with no oscillations. 
However the elastic mismatch between the elastic constants of perfectly bonded interfaces or artificial "non-perfect" conditions on the interface are a mere idealization of complex phenomena at the interface and does not completely describe the structural and chemical characteristics of the interface separating both media. The corollary of the above assertion is that any theoretical framework describing interface crack problems needs to not only include the mismatch between the elastic constants but also the interfacial structural mismatch accounting for the effects of the interface structure (both cracked and uncracked regions) and its associated elastic behavior.

In this manuscript, the two-dimensional (2-D) problem of an interface crack between two anisotropic elastic solids is considered. The interfacial structure and state of coherency at the interface is accounted for by means of an interfacial elasticity paradigm (Section 2). This interface model introduces boundary conditions that are effectively equivalent to those for a weakly bounded interfaces (Appendix B). Using these boundary conditions, the elastic fields (i.e., displacements and stresses) in the vicinity of a crack are derived based on the Stroh formalism (Section 3). A discussion on the order of the singularity and the oscillatory effects appearing in problems involving interface cracks concludes this manuscript (Section 4).

A list of notation and convention used throughout this manuscript is cataloged in Appendix A.

\section{Basic equations of elasticity considering surface elasticity}

Consider two dissimilar semi-infinite linear elastic anisotropic half spaces having stiffness elasticity tensors $\mathbb{C}^{+}$and $\mathbb{C}^{-}$and being separated by an interface $S$ (located at $x_{2}=0$ ). Medium "+" and medium "-_" are defined for $x_{2}>0$ and $x_{2}<0$ respectively. For convenience and clarity, all the field variables associated with the problem addressed here are confined to the two-dimensional space $x_{1}-x_{2}$. If we denote the displacement, strain and stress fields by $\boldsymbol{u}, \boldsymbol{\epsilon}$, and $\boldsymbol{\sigma}$, respectively, the Hooke's law in general anisotropy reads,

$$
\sigma_{i j}=\mathbb{C}_{i j k l} \epsilon_{k l}^{e l}
$$

where Roman indices range from 1 to 3 and Greek indices are taken as either 1 or 3 (i.e. in the plane of the interface), unless otherwise indicated. A comma followed by an index indicates the partial differentiation with respect to the corresponding coordinate variable and repeated indices are summed.

This bimaterial is subjected to a homogeneous traction and displacement boundary condition at infinity, such that far away from the interface the deformation can be assumed homogeneous, and the transverse stress and the in-plane strain tensors 
in the upper $\left(S_{+}\right)$and lower $\left(S_{-}\right)$materials are given by $\boldsymbol{\sigma}^{\perp, \infty}=\left(\sigma_{21}^{\infty}, \sigma_{22}^{\infty}, \sigma_{23}^{\infty}\right)$ and $\boldsymbol{\epsilon}^{S, \infty}=\left(\epsilon_{11}^{\infty}, \epsilon_{33}^{\infty}, 2 \epsilon_{13}^{\infty}\right)$ respectively. It has been shown that such homogeneous deformation can be easily constructed in a bimaterial (Qu and Bassani, 1993) by the so-called "T-decomposition".

Following Dingreville et al. (2014), the in-plane strain $\boldsymbol{\epsilon}^{S}$ assumes continuity of displacement along the interface i.e., $\boldsymbol{\epsilon}^{S}=\boldsymbol{\epsilon}_{+}^{S}=\boldsymbol{\epsilon}_{-}^{S}$. Additionally, as described in more detail in Section 3, a second strain measure in the form of a coherency eigenstrain $\epsilon^{*, S}$ is considered. This strain measure is associated with the interface to represent the state of coherency between the two solids (Dingreville et al., 2014). It describes the relaxation of lattice mismatches resulting in the formation of peculiar interfacial structures. For a coherent interface, the mismatch is completely accommodated by straining both phases. In the case of a semi-coherent interface, localized misfit dislocations are assumed to be responsible for compensating uniform far-field elastic fields, while an incoherent interface is the result of two rigid semi-infinite media in rigid contact (Romanov et al., 1998; Romanov and Wagner, 2001).

\subsection{Lekhnitskii-Eshelby-Stroh (LES) representation in 2-D anisotropic elasticity}

Problems of two-dimensional anisotropic elasticity as described above can be treated by the so-called Lekhnitskii-Eshelby-Stroh (LES) formalism (Eshelby et al., 1953; Stroh, 1958; Lekhnitskii, 1963). This approach can be considered as an extension of the stress function used in isotropic elasticity for anisotropic problems. The Navier equation governing the elasticity solutions is given by,

$$
\mathbb{C}^{ \pm}: \nabla \cdot \nabla \boldsymbol{u}_{ \pm}(\mathbf{x})=\mathbb{C}^{ \pm}: \nabla \boldsymbol{\epsilon}_{ \pm}^{*, S}(\mathbf{x})
$$

where $\boldsymbol{u}_{ \pm}(\mathbf{x})$ is the displacement field in medium "+" and "-" respectively. To avoid confusion and cumbersome notation, the subscripts \pm are ignored in the remainder of this section. Leveraging the two-dimensionality of the problem, the Navier equation (2) can be rewritten in the matrix form,

$$
\boldsymbol{Q} \frac{\partial^{2} \boldsymbol{u}}{\partial x_{1}^{2}}\left(x_{1}, x_{2}\right)+\left[\boldsymbol{R}+\boldsymbol{R}^{T}\right] \frac{\partial^{2} \boldsymbol{u}}{\partial x_{1} \partial x_{2}}\left(x_{1}, x_{2}\right)+\boldsymbol{T} \frac{\partial^{2} \boldsymbol{u}}{\partial x_{2}^{2}}\left(x_{1}, x_{2}\right)=\varpi^{*}\left(x_{1}, x_{2}\right),
$$

where the superscript $T$ denotes the transpose. The real matrices $\boldsymbol{Q}, \boldsymbol{R}$ and $\boldsymbol{T}$ are obtained from the elastic stiffness tensor such that,

$$
Q_{i k}=\mathbb{C}_{i 1 k 1}, R_{i k}=\mathbb{C}_{i 1 k 2}, T_{i k}=\mathbb{C}_{i 2 k 2},
$$

and the virtual coherency body forces vector $\varpi^{*}$ is a function of the coherency eigenstrain such that,

$$
\varpi_{i}^{*}=\mathbb{C}_{i j k l} \epsilon_{k l, j}^{*, S}
$$


Note that properties (Hermitian, singularity, definiteness) of the matrices $\boldsymbol{Q}, \boldsymbol{T}, \boldsymbol{R}$ and their subsequent manipulations are related to the physicality of the problem (strain energy, elasticity constants, etc.) and the existence of solutions.

Through superposition, the solution to the Poisson's equation (3) is the sum of a homogeneous and particular solution. The expression of the general solution $\boldsymbol{u}\left(x_{1}, x_{2}\right)$ is determined from the homogeneous boundary value problem $\boldsymbol{u}^{0}\left(x_{1}, x_{2}\right)$ and the boundary conditions (sub-section 2.1.2), while the particular solution $\boldsymbol{u}^{*}\left(x_{1}, x_{2}\right)$ derives from the right side of the partial differential equation, i.e., the virtual coherency body forces (sub-section 2.1.1).

\subsubsection{Solution to the particular eigenvalue problem}

Let us start with the particular solution corresponding to the virtual coherency body forces introduced by the coherency eigenstrain $\boldsymbol{\epsilon}^{*, S}$. Assuming $\boldsymbol{\epsilon}^{*, S}$ only varies in the direction transverse to the interface, the particular solution $\boldsymbol{u}^{*}$ to the Navier equation (Eq. (2)) can be easily obtained by applying the Fourier transform of the displacement vector with respect to $x_{2}$, represented by $\tilde{\boldsymbol{u}}\left(x_{1}, \xi\right)$, such that Eq. (3) becomes in the Fourier space,

$$
\boldsymbol{Q} \frac{\partial^{2} \tilde{\boldsymbol{u}}}{\partial x_{1}^{2}}\left(x_{1}, \xi\right)-\mathrm{i} \xi\left[\boldsymbol{R}+\boldsymbol{R}^{T}\right] \frac{\partial \tilde{\boldsymbol{u}}}{\partial x_{1}}\left(x_{1}, \xi\right)-\xi^{2} \boldsymbol{T} \tilde{\boldsymbol{u}}\left(x_{1}, \xi\right)=\tilde{\boldsymbol{\varpi}}^{*}(\xi)
$$

with,

$$
\begin{aligned}
\tilde{\boldsymbol{u}}\left(x_{1}, \xi\right) & =\frac{1}{\sqrt{2 \pi}} \int_{-\infty}^{\infty} \boldsymbol{u}\left(x_{1}, x_{2}\right) e^{\mathrm{i} \xi x_{2}} d x_{2}, \\
\tilde{\varpi}^{*}(\xi) & =\frac{1}{\sqrt{2 \pi}} \int_{-\infty}^{\infty} \varpi^{*}\left(x_{2}\right) e^{\mathrm{i} \xi x_{2}} d x_{2},
\end{aligned}
$$

and $\mathrm{i}=\sqrt{-1}$. Consequently, the expressions of the particular solution $\tilde{\boldsymbol{u}}^{*}$ and $\boldsymbol{u}^{*}$, in both the Fourier and the real spaces, are given by the two following equations,

$$
\begin{aligned}
\tilde{\boldsymbol{u}}^{*}\left(x_{1}, \xi\right) & =-\boldsymbol{T}^{-1} \xi^{-2} \tilde{\varpi}^{*}(\xi) \\
\boldsymbol{u}^{*}\left(x_{1}, x_{2}\right) & =-\frac{\boldsymbol{T}^{-1}}{\sqrt{2 \pi}} \int_{-\infty}^{\infty} \xi^{-2} \tilde{\varpi}^{*}(\xi) e^{-\mathrm{i} \xi x_{2}} d \xi
\end{aligned}
$$

Note that for the case in which the coherency eigenstrain tensor depends on both $x_{1}$ and $x_{2}$, the same method can be applied except that the Fourier transform will have to be performed with respect to both $x_{1}$ and $x_{2}$. 


\subsubsection{Solution to the homogeneous eigenvalue problem}

For the homogeneous problem (i.e., $\varpi^{*}=0$ ), it is assumed that the displacements $\boldsymbol{u}^{0}\left(x_{1}, x_{2}\right)$ have the form (Stroh, 1958, 1962),

$$
\boldsymbol{u}^{0}\left(x_{1}, x_{2}\right)=\boldsymbol{a} f(z) \text { with } z=x_{1}+p x_{2} .
$$

The function $f(z)$ is an arbitrary analytic function, while the vector $\boldsymbol{a}$ and the complex variable $p$ satisfy the following eigenvalue equation,

$$
\left(\boldsymbol{Q}+p\left[\boldsymbol{R}+\boldsymbol{R}^{T}\right]+p^{2} \boldsymbol{T}\right) \boldsymbol{a}=\mathbf{0} .
$$

A non-trivial solution to Eq. (12) requires its determinant to be zero. It has been proven that all roots of Eq. (12) consist of three pairs of complex conjugate roots $\left\{p_{i}\right\}$ as a consequence of the positive definiteness of the strain energy (Eshelby et al., 1953). Additionally the following classical convention is used,

$$
p_{i}=\bar{p}_{i+3}, \quad \mathfrak{I m}\left(p_{i}\right)>0, i=\{1,2,3\},
$$

where the over-bar denotes the complex conjugate and the notation $\mathfrak{I m}($ ) denotes the imaginary part of a complex number.

The general solution for the displacement and stress function vector $\boldsymbol{\Phi}^{0}$ can then be written as,

$$
\begin{aligned}
u_{i}^{0} & =2 \mathfrak{R e}\left(A_{i j} f_{j}\left(z_{j}\right)\right), \\
\Phi_{i}^{0} & =2 \mathfrak{R e}\left(B_{i j} f_{j}\left(z_{j}\right)\right),
\end{aligned}
$$

where

$$
z_{j}=x_{1}+p_{j} x_{2}
$$

and the notation $\mathfrak{R e}($ ) is the real part of a complex number. The matrices $\boldsymbol{A}$ and $\boldsymbol{B}$ in Eqs. (14) and (15) are two $3 \times 3$ matrices depending on the elastic constants given by,

$$
\begin{aligned}
\boldsymbol{A} & =\left[\boldsymbol{a}_{1}, \boldsymbol{a}_{2}, \boldsymbol{a}_{3}\right] \\
\boldsymbol{B} & =\boldsymbol{R}^{T} \boldsymbol{A}+\boldsymbol{T} \boldsymbol{A}\left\langle p_{*}\right\rangle=-\left(\boldsymbol{Q} \boldsymbol{A}+\boldsymbol{R} \boldsymbol{A}\left\langle p_{*}\right\rangle\right)\left\langle p_{*}^{-1}\right\rangle,
\end{aligned}
$$

with the diagonal matrix $\left\langle p_{*}\right\rangle=\operatorname{diag}\left[p_{1}, p_{2}, p_{3}\right]$.

Using Hooke's law, the transverse and in-plane stresses are related to the stress function vector $\Phi^{0}$ by,

$$
\begin{aligned}
\boldsymbol{\sigma}^{\perp} & =\boldsymbol{\Phi}_{, 1}^{0}=2 \mathfrak{R e}\left(B_{i j} f_{j}^{\prime}\left(z_{j}\right)\right), \\
\boldsymbol{\sigma}^{S} & =-\boldsymbol{\Phi}_{, 2}^{0}=-2 \mathfrak{R e}\left(B_{i j} p_{j} f_{j}^{\prime}\left(z_{j}\right)\right),
\end{aligned}
$$


with $\sigma_{i}^{\perp}=\sigma_{i 2}$ and $\sigma_{i}^{S}=\sigma_{i 1}$. The function $f^{\prime}$ designates the derivative of the function $f$ with respect to its associated argument.

Equations (14), (19), and (20) completely represent the displacement and stress fields. This is done by solving the function vector $\boldsymbol{f}=\left[f_{1}\left(z_{1}\right), f_{2}\left(z_{2}\right), f_{3}\left(z_{3}\right)\right]^{T}$ by considering the proper boundary conditions (accounting for interfacial elasticity in the present work). Matrices $\boldsymbol{A}$ and $\boldsymbol{B}$ play an important role with respect to interfacial crack problems and certain aspects of their mathematical properties need to be noted. Indeed, it has been shown (Stroh, 1958) that $\boldsymbol{A}$ and $\boldsymbol{B}$ are non-singular when the six complex roots are all distinct or for some specific non-degenerate cases (Ting and $\mathrm{Hwu}, 1988)$. Additional details regarding the degenerate and non-degenerate cases can be found elsewhere (Ting, 1996, 2000). Furthermore, the product $\boldsymbol{A B}^{-1}$ is anti-Hermitian (i.e., $\left[\boldsymbol{A} \boldsymbol{B}^{-1}\right]^{T}=-\overline{\boldsymbol{A B}}^{-1}$ ). By calculating the energy of a line force acting on an anisotropic space, Chadwick and Smith (1977) proved that i $\boldsymbol{A B}^{-1}$ is Hermitian and positive definite. Finally, both of the matrices $\boldsymbol{A}$ and $\boldsymbol{B}$ and the eigenvalues $p_{i}$ are complex quantities depending on the various elastic moduli. We will make use of these properties when studying various interfacial problems. Given those properties, it is assumed that in the remainder of this manuscript, both materials fulfill the condition of distinct and non-singular complex eigenvalues (i.e. the existence of three independent eigenvectors).

\subsection{Boundary conditions associated with interface region considering interfacial elas- ticity}

The inclusion of the interface structure and its mechanical behavior through interfacial elasticity yields non-classical boundary conditions. The boundary conditions used to solve the interfacial crack problem are based a generalized interfacial elasticity formulation (Dingreville et al., 2014) describing the elastic behavior of coherent and incoherent interfaces. This generalized Shuttleworth-Herring formulation considers the equilibrium of mismatched interfaces as a function of the in-plane strain $\boldsymbol{\epsilon}^{S}$, the transverse stress $\boldsymbol{\sigma}^{\perp}$ and the structural mismatch strain $\boldsymbol{\epsilon}^{m, S}$. This formulation not only accounts for the three-dimensional nature of the interface in a Gibbsian sense but also explicitly considers its interfacial structure. Within this formalism, a coherent surface stress $\Sigma^{S}$ and a transverse excess strain $\boldsymbol{\Delta}^{\perp}$ are introduced. The coherent surface stress $\boldsymbol{\Sigma}^{S}$ and the transverse excess strain $\boldsymbol{\Delta}^{\perp}$ represent the thermodynamic driving forces associated with the deformation of the interface. These quantities characterize the elastic behavior of coherent and incoherent interfaces and 
read,

$$
\begin{aligned}
\boldsymbol{\Sigma}^{S} & =\left.\frac{\partial \Gamma}{\partial \boldsymbol{\epsilon}^{S}}\right|_{\boldsymbol{\epsilon}^{m, S}, \boldsymbol{\sigma}^{\perp}}+\boldsymbol{\sigma}^{\perp} \cdot \mathbb{H}^{S} \\
\boldsymbol{\Delta}^{\perp}-\boldsymbol{\Lambda}_{0}^{\perp} & =\left.\frac{\partial \Gamma}{\partial \boldsymbol{\sigma}^{\perp}}\right|_{\boldsymbol{\epsilon}^{S}, \boldsymbol{\epsilon}^{m, S}}-\mathbb{H}^{S}: \boldsymbol{\epsilon}^{S}+\mathbb{K}^{S}: \boldsymbol{\epsilon}^{m, S} .
\end{aligned}
$$

The constitutive behavior of the interface is derived from the interfacial excess free energy $\Gamma$ in the form,

$$
\begin{aligned}
\Gamma=\hat{\Gamma}\left(\boldsymbol{\epsilon}^{S}, \boldsymbol{\epsilon}^{m, S}, \boldsymbol{\sigma}^{\perp}\right) & =\Gamma_{0}+\boldsymbol{\Sigma}_{0}^{S}: \boldsymbol{\epsilon}^{S}+\frac{1}{2} \boldsymbol{\epsilon}^{S}: \mathbb{C}^{S}: \boldsymbol{\epsilon}^{S}+\frac{1}{2} \boldsymbol{\sigma}^{\perp} \cdot \boldsymbol{\Lambda}^{\perp} \cdot \boldsymbol{\sigma}^{\perp} \\
& +\Upsilon_{0}^{S}: \boldsymbol{\epsilon}^{m, S}+\frac{1}{2} \boldsymbol{\epsilon}^{m, S}: \boldsymbol{\Upsilon}_{2}^{S}: \boldsymbol{\epsilon}^{m, S}-\boldsymbol{\epsilon}^{m, S}: \boldsymbol{\Phi}^{S}: \boldsymbol{\epsilon}^{S},
\end{aligned}
$$

Consequently, the coherent surface stress and interfacial strain constitutive equations read,

$$
\boldsymbol{\Sigma}^{S}=\boldsymbol{\Sigma}_{0}^{S}-\boldsymbol{\Phi}^{S}: \boldsymbol{\epsilon}^{m, S}+\mathbb{C}^{S}: \boldsymbol{\epsilon}^{S}+\boldsymbol{\sigma}^{\perp} \cdot \mathbb{H}^{S}
$$

and,

$$
\boldsymbol{\Delta}^{\perp}=\boldsymbol{\Lambda}_{0}^{\perp}+\mathbb{K}^{S}: \boldsymbol{\epsilon}^{m, S}+\boldsymbol{\Lambda}^{\perp} \cdot \boldsymbol{\sigma}^{\perp}-\mathbb{H}^{S}: \boldsymbol{\epsilon}^{S} .
$$

The three surface elastic tensors $\left(\mathbb{C}^{S}, \Lambda^{\perp}, \mathbb{H}^{S}\right)$ describe the pure elastic behavior of the interface (i.e., independent of the structural mismatch). The four surface coupling tensors $\left(\Sigma_{0}^{S}, \boldsymbol{\Lambda}_{0}^{\perp}, \boldsymbol{\Phi}^{S}, \mathbb{K}^{S}\right)$ describe and/or account for the coupling between the interface and the state of coherency (i.e., dependent on the structure of the interface). The two coherency tensors $\Upsilon_{0}^{S}$ and $\Upsilon_{2}^{S}$ describe the state of coherency of the interface while the scalar $\Gamma_{0}$ represents the residual interfacial excess energy. All of these tensors are intrinsic properties of the interface. More details are provided in Dingreville et al. (2014) for a complete explanation of the various terms.

Note that $\boldsymbol{\Sigma}^{S}$ and $\boldsymbol{\Delta}^{\perp}$ are the only thermodynamic quantities necessary to describe the mechanical equilibrium conditions on the interface. Although not presented here, an incoherent stress $\mathbf{\Upsilon}^{S}$ is also introduced within this formalism. Subsequently, the interfacial conditions across the interface (see Appendix B derivation) consist of the following equations,

$$
\begin{aligned}
\llbracket \mathbf{u} \rrbracket & =\boldsymbol{\Delta}^{\perp} \\
\mathbf{n} \cdot \llbracket \boldsymbol{\sigma} \rrbracket \cdot \mathbf{n} & =-\boldsymbol{\Sigma}^{S}: \boldsymbol{\kappa}, \\
\mathbf{P}^{S} \cdot \llbracket \boldsymbol{\sigma} \rrbracket \cdot \mathbf{n} & =-\nabla^{S} \boldsymbol{\Sigma}^{S} .
\end{aligned}
$$

The bracket symbol $\llbracket \cdot \rrbracket$ for any function $\phi$ denotes the jump across the interface such that $\llbracket \phi \rrbracket=\phi\left(x_{1}, 0^{+}\right)-\phi\left(x_{1}, 0^{-}\right)$. The tensor $\boldsymbol{\kappa}$ is the curvature tensor, the 
tensor $\mathbf{P}^{S}$ is the surface projection tensor, and $\nabla^{S}$ is surface nabla operator (see definitions in Appendix B). Note that these boundary conditions are equivalent to those of Gurtin and Murdoch (1975) when the transverse behavior of the dividing surface is neglected. Thus, this interfacial elasticity formulation introduces boundary conditions that are effectively equivalent to those of a weakly bounded interface (also defined as "seemingly imperfectly bonded" in the remainder of this manuscript). The jump in traction across the interface is related to the coherent surface stress $\Sigma^{S}$ and the interface curvature. The jump in displacement is related to the transverse excess strain $\boldsymbol{\Delta}^{\perp}$. Tensor $\boldsymbol{\Delta}^{\perp}$ can be physically interpreted as an intrinsic thickness of the interface. Note both of these jump conditions are pure artifacts of the Gibbsian description of the interface and the associated vanishing thickness of the interfacial region.

\section{Interfacial Griffith crack with surface elasticity in an anisotropic bima- terial}

\subsection{Problem formulation}

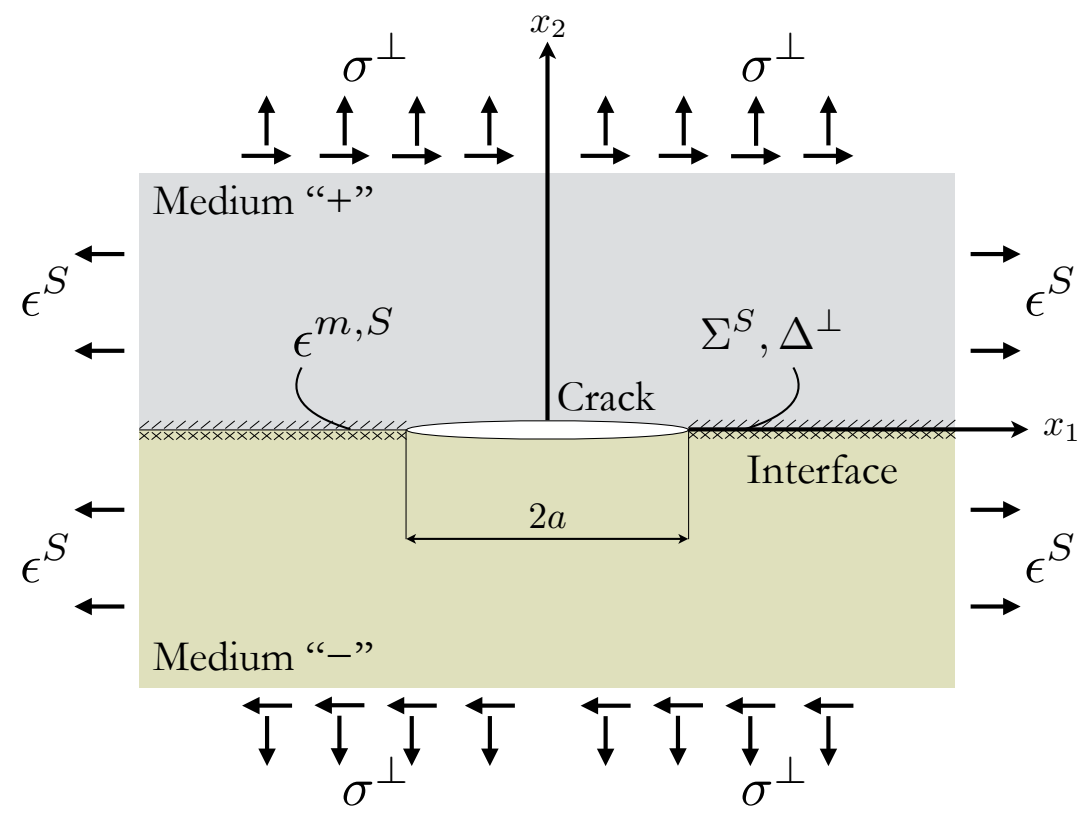

Figure 1: An interface Griffith crack embedded between two dissimilar elastic half-planes. Interface properties are considered through an interfacial elasticity formulation 
As illustrated in Fig. 1, a Griffith crack of length $L=2 a$ is considered along an incoherent planar interface $S$ between the two semi-infinite media. The crack tips lie along the interface $x_{2}=0$ for $\left|x_{1}\right| \leq a$. The interface is being strained by a homogeneous strain $\boldsymbol{\epsilon}^{S, \infty}$ in the plane of the interface $\left(x_{1}\right.$-axis) and loaded transversely ( $x_{2}$-axis) by a remote stress $\boldsymbol{\sigma}^{\perp, \infty}$ ("T-decomposition"). Additionally, following Cahn and Larché (1982) and Dingreville et al. (2014), a coherency eigenstrain $\boldsymbol{\epsilon}^{*, S}$ defined only in medium "-" (medium "+" is arbitrarily chosen as the reference state) is introduced to account for the interface incoherency and interface structure. The strain tensor $\boldsymbol{\epsilon}^{*, S}$ is decomposed into two components (Dingreville et al., 2014) such that,

$$
\boldsymbol{\epsilon}^{*, S}(\mathbf{x})=\left[\boldsymbol{\epsilon}^{0, S}+\boldsymbol{\epsilon}^{m, S} g(\mathbf{x})\right] H\left(-x_{2}\right),
$$

where $H\left(-x_{2}\right)$ denotes the Heaviside function. The strain tensor $\boldsymbol{\epsilon}^{0, S}$ is an eigenstrain related to the change in molar volume between medium "+" and medium "_" while $\boldsymbol{\epsilon}^{m, S}$ represents the in-plane interfacial structural mismatch at the interface (i.e., $x_{2}=0$ ). The spatial function $g(\mathbf{x})$ defined in Eq. (29) corresponds to the variation of this structural mismatch over few atomic layers and vanishes far from the interface. As such, $g\left(x_{1}, 0\right)=1$ by definition of the in-plane structural mismatch $\boldsymbol{\epsilon}^{m, S}$, and $g\left(x_{1}, x_{2}\right)=0, \forall x_{2} \leq-h$, where $h$ is an arbitrary distance far away from the interface. The structural mismatch at the interface is usually defined as (Romanov et al., 1998),

$$
\epsilon_{\alpha \beta}^{m, S}=2 \frac{\ell_{+}-\ell_{-}}{\ell_{+}+\ell_{-}} \delta_{\alpha \beta},
$$

where $\ell_{+}$and $\ell_{-}$are the crystal lattice parameters in medium + and - respectively and $\delta_{\alpha \beta}$ is the Kronecker symbol.

As mentioned earlier, the solution to Eq. (2) consists of the sum of the homogeneous and particular solutions. The existence of the particular solution is solely due to the existence of the coherency eigenstrain $\epsilon^{*, S}$ in the lower half-space $S_{-}$ which accounts for both the interface incoherency and interface structure. The physical problem associated with the homogeneous solution consists of a finite crack lying between two distinct and seemingly imperfectly bonded materials subjected to a uniform loading at infinity. Following England (2003) and as illustrated in Fig. 2, the problem defined above is equivalent to the superposition of three simpler problems:

1. The first problem (Section 3.2) corresponds to particular solution of an uncracked bimaterial of two perfectly bonded anisotropic materials subjected to the coherency eigenstrain $\boldsymbol{\epsilon}^{*, S}$.

2. The second problem (Section 3.3) consists of an uncracked bimaterial of two seemingly imperfectly bonded (in the context of the interfacial boundary conditions) anisotropic materials and subjected to known stresses at infinity which, 
combined with the boundary conditions along the interface, brings about a traction distribution $-\mathbf{t}^{*}(\mathbf{x})$ across the crack segment $[-a, a]$.

3. The third problem (Section 3.4) considers the classical problem of a bimaterial containing a crack lying along a perfectly bonded interface. No loading is applied at infinity but the crack is being opened by the traction force $\mathbf{t}^{*}(\mathbf{x})$ obtained from the second problem and prescribed on the crack faces; the resultant force over the crack faces is equal to zero. In other words, the traction vectors on the upper and lower surface of the cracks are therefore denoted as $\mathbf{t}^{*}(\mathbf{x})$ and $-\mathbf{t}^{*}(\mathbf{x})$ respectively.

It should be mentioned that in the third problem, for the sake of clarity and avoid any confusion, we chose not to consider surface stresses (in the Gibbsean sense) on the crack faces. As mentioned earlier, such developments have been considered by others (Feng et al., 2008; Antipov and Schiavone, 2011; Kim et al., 2011; Nan and Wang, 2012; Koguchi and Suzuki, 2014; Wang et al., 2015; Sigaeva and Schiavone, 2016). Surface stresses for free surfaces of the crack faces may be on the same order of magnitude as the interface stresses outside of the crack segment but their constitutive relationships differ from the ones introduced in Eqs. (24)-(25). The added complexity only impacts solutions to the third problem and the reader is referred to the work mentioned above for additional details on that topic.

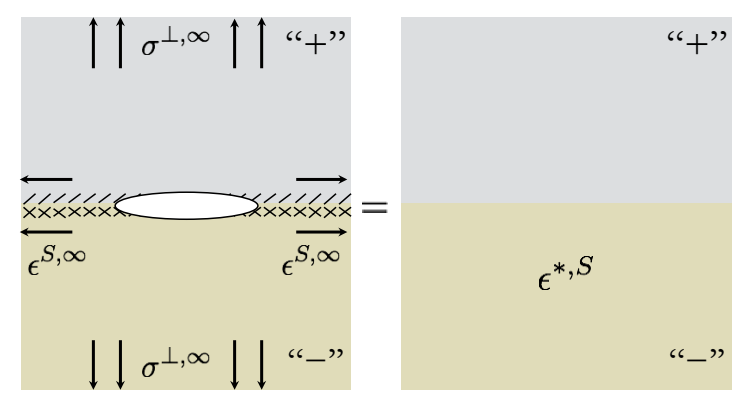

(a)

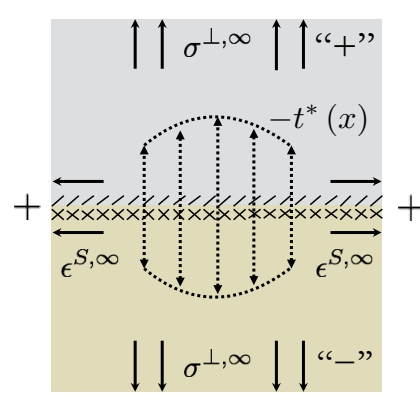

(b)

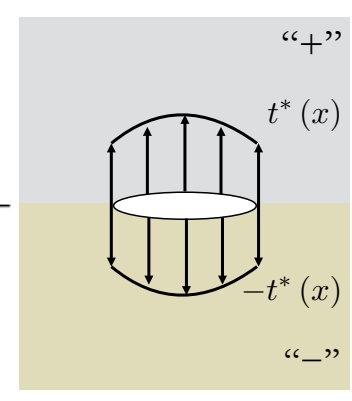

(c)

Figure 2: An interface Griffith crack between two dissimilar elastic half-planes. (a) A coherency eigenstrain considered in medium "-". (b) Elastic fields for a seemingly imperfectly bonded interface. (c) An interface crack with traction prescribed on the faces.

\subsection{Particular solution to the coherency eigenstrain $\boldsymbol{\epsilon}^{*, S}$}

We first consider the uncracked bimaterial of two perfectly bonded anisotropic materials subjected to the coherency eigenstrain $\boldsymbol{\epsilon}^{*, S}$. Starting from Eq. (29), the 
function $g(\mathbf{x})$ is assumed to be a continuous function taking the generic form,

$$
g\left(x_{2}\right)=\left\{\begin{array}{r}
0,-\infty<x_{2}<-h \\
g^{*}\left(x_{2}\right),-h \leq x_{2}<0 \\
1, x_{2} \geq 0
\end{array}\right.
$$

where the function $g^{*}$ is also a continuous function over the domain $[-h, 0]$, such that $g^{*}(-h)=0$ and $g^{*}(0)=1$. Substituting Eq. (31) into Eq. (5) yields,

$$
\varpi_{i}^{*}\left(x_{2}\right)=-\mathbb{C}_{i 2 k l}\left[\epsilon_{k l}^{0, S}+\epsilon_{k l}^{m, S} g\left(x_{2}\right)\right] \delta\left(-x_{2}\right)+\mathbb{C}_{i 2 k l} \epsilon_{k l}^{m, S} \frac{\partial g\left(x_{2}\right)}{\partial x_{2}} H\left(-x_{2}\right),
$$

or equivalently,

$$
\varpi_{i}^{*}\left(x_{2}\right)=R_{k i}\left[\epsilon_{k 1}^{m, S} \varphi\left(x_{2}\right)-\epsilon_{k 1}^{0, S} \delta\left(x_{2}\right)\right]-T_{i k} \epsilon_{k 2}^{0, S} \delta\left(x_{2}\right),
$$

with, $\varphi\left(x_{2}\right)=g^{\prime}\left(x_{2}\right) H\left(-x_{2}\right)-g\left(x_{2}\right) \delta\left(x_{2}\right)$. Substituting Eq. (33) into Eq. (10) simply gives the displacements $u_{i}^{*}\left(x_{1}, x_{2}\right)$ in the real space such that,

$$
u_{i}^{*}\left(x_{1}, x_{2}\right)=u_{i}^{0, *}\left(x_{2}\right)-\frac{T_{i j}^{-1} R_{k j} \epsilon_{k 1}^{m, S}}{\sqrt{2 \pi}} \int_{-\infty}^{\infty} \tilde{\varphi}(\xi) \xi^{-2} e^{-\mathrm{i} \xi x_{2}} d \xi,
$$

with,

$$
\tilde{\varphi}(\xi)=\frac{1}{\sqrt{2 \pi}} \int_{-h}^{0} g^{\prime}\left(x_{2}\right) e^{\mathrm{i} x_{2} \xi} d x_{2}-\frac{g(0)}{\sqrt{2 \pi}}
$$

and,

$$
u_{i}^{0, *}\left(x_{2}\right)=-\left[T_{i j}^{-1} R_{k j} \epsilon_{k 1}^{0, S}+\epsilon_{i 2}^{0, S}\right] \frac{x_{2}}{2} \operatorname{sgn}\left(x_{2}\right) .
$$

Depending on the functional form for $g^{*}$, Eq. (34) can be carried analytically or numerically.

The associated stress field can then simply be obtained through Hooke's law such that,

$$
\sigma_{i j}^{*}\left(x_{1}, x_{2}\right)=\sigma_{i j}^{0, *}\left(x_{2}\right)-\frac{\mathbb{C}_{i j k 2} T_{k p}^{-1} R_{l p} \epsilon_{l 1}^{m, S}}{\sqrt{2 \pi}} \frac{\partial}{\partial x_{2}} \int_{-\infty}^{\infty} \tilde{\varphi}(\xi) \xi^{-2} e^{-\mathrm{i} \xi x_{2}} d \xi
$$

with,

$$
\begin{aligned}
\sigma_{i j}^{0, *}\left(x_{2}\right) & =-\mathbb{C}_{i j k 2}\left(T_{k p}^{-1} R_{l p} \epsilon_{l 1}^{0, S}+\epsilon_{k 2}^{0, S}\right)\left(\frac{\operatorname{sgn}\left(x_{2}\right)}{2}+x_{2} \delta\left(x_{2}\right)\right) \\
& -\mathbb{C}_{i j k l}\left(\epsilon_{k l}^{0, S}+\epsilon_{k l}^{m, S} g\left(x_{2}\right)\right) H\left(-x_{2}\right)
\end{aligned}
$$


Equations (34) and (37) clearly show the strong dependency on the coupling between the bulk elastic constants and the two tensors $\boldsymbol{\epsilon}^{0, S}$ and $\boldsymbol{\epsilon}^{m, S}$. However, one of the main differences between the present formulation and others such as the one proposed by Koguchi and Hirasawa (2015) for example, lies in the existence of the coherency eigenstrain tensor $\epsilon^{*, S}$ solely in $S_{-}$. Both approaches account for the coherency of the interface via the interface boundary conditions but the present formulation describes the transition between materials "+" and "-" as a smooth process occurring over a few atomic layers (see definition of the function $g$ ) as opposed to a displacement and/or stress discontinuity across the $x_{2}$-axis (Koguchi and Hirasawa, 2015).

\subsection{Solution for imperfectly bonded anisotropic bimaterials}

We now consider the second problem consisting of two anisotropic materials separated by a seemingly imperfect interface characterized by the interfacial elasticity boundary conditions given in Eqs. (26)-(28). The prescribed loading consists of transverse stresses $\boldsymbol{\sigma}^{\perp, \infty}=\left(\sigma_{21}^{\infty}, \sigma_{22}^{\infty}, \sigma_{23}^{\infty}\right)$ and in-plane strains $\boldsymbol{\epsilon}^{S, \infty}=\left(\epsilon_{11}^{\infty}, \epsilon_{33}^{\infty}, 2 \epsilon_{13}^{\infty}\right)$, both applied at infinity. The second term of the in-plane strain tensor is set to zero.

Based on the Stroh formalism and considering the boundary conditions at infinity (Hwu, 2010), the components of the displacement vector and stress function at infinity in $S_{+}$and $S_{-}$can be represented by selecting the complex function vector $\mathbf{f}(z)=\left\langle z_{\alpha}^{i}\right\rangle \mathbf{q}$ such that,

$$
\begin{aligned}
u_{m}^{\infty} & \propto 2 \mathfrak{R e}\left(\boldsymbol{A}_{m}\left\langle z_{\alpha_{m}}\right\rangle\left[\boldsymbol{A}_{m}^{T} \boldsymbol{g}_{\sigma}+\boldsymbol{B}_{m}^{T} \boldsymbol{h}_{\epsilon}\right]\right) \\
\Phi_{m}^{\infty} & \propto 2 \mathfrak{R e}\left(\boldsymbol{B}_{m}\left\langle z_{\alpha_{m}}\right\rangle\left[\boldsymbol{A}_{m}^{T} \boldsymbol{g}_{\sigma}+\boldsymbol{B}_{m}^{T} \boldsymbol{h}_{\epsilon}\right]\right)
\end{aligned}
$$

where the complex coefficient vector $\mathbf{q}$ has been replaced by two real constant vectors $\boldsymbol{g}_{\sigma}$ and $\boldsymbol{h}_{\epsilon}$ reflecting the T-decomposition, $\left[\boldsymbol{h}_{\epsilon}, \boldsymbol{g}_{\sigma}\right]^{T}=\left[\boldsymbol{\epsilon}_{1}^{\infty}, \boldsymbol{\sigma}^{\perp, \infty}\right]^{T}, \boldsymbol{\epsilon}_{1}^{\infty}=$ $\left[\epsilon_{11}^{\infty}, \epsilon_{12}^{\infty}, 2 \epsilon_{13}^{\infty}\right]^{T}$ and $m=\{+,-\}$. The following identities (Ting, 1986) should be noted as they will be used later on in the derivations:

$$
\begin{aligned}
2 \mathfrak{R e}\left(\boldsymbol{A}_{m}\left[\boldsymbol{A}_{m}^{T} \boldsymbol{g}_{\sigma}+\boldsymbol{B}_{m}^{T} \boldsymbol{h}_{\epsilon}\right]\right) & =\boldsymbol{h}_{\epsilon} \\
2 \mathfrak{R e}\left(\boldsymbol{B}_{m}\left[\boldsymbol{A}_{m}^{T} \boldsymbol{g}_{\sigma}+\boldsymbol{B}_{m}^{T} \boldsymbol{h}_{\epsilon}\right]\right) & =\boldsymbol{g}_{\sigma} .
\end{aligned}
$$

Traction continuity condition: Considering the traction continuity across the interface (for a flat interface $\boldsymbol{\kappa}=0$, therefore $\mathbf{n} \cdot \llbracket \boldsymbol{\sigma} \rrbracket \cdot \mathbf{n}=0$ ) for the whole $x_{1}$-axis, Eq. (27) yields,

$$
\boldsymbol{B}_{+} \boldsymbol{f}_{+}^{\prime}\left(x_{1}\right)-\overline{\boldsymbol{B}_{-}} \overline{\boldsymbol{f}_{-}^{\prime}}\left(x_{1}\right)=\boldsymbol{B}_{-} \boldsymbol{f}_{-}^{\prime}\left(x_{1}\right)-\overline{\boldsymbol{B}_{+}} \overline{\boldsymbol{f}_{+}^{\prime}}\left(x_{1}\right) .
$$

In order to simplify subsequent derivations, it is useful to define a complex potential $\boldsymbol{\theta}(z)$ which is analytic (i.e. locally given by a convergent power series) in the 
whole complex plane, including points at infinity. By using the method of analytical continuation (Suo, 1990; England, 2003), $\boldsymbol{\theta}(z)$ can be defined by,

$$
\boldsymbol{\theta}(z)= \begin{cases}\boldsymbol{B}_{+} \boldsymbol{f}_{+}^{\prime}(z)-\overline{\boldsymbol{B}_{-}} \overline{\boldsymbol{f}_{-}^{\prime}}(z), z \in S_{+} \\ \boldsymbol{B}_{-} \boldsymbol{f}_{-}^{\prime}(z)-\overline{\boldsymbol{B}_{+}} \overline{\boldsymbol{f}_{+}^{\prime}}(z), z \in S_{-}\end{cases}
$$

According to the Liouville's theorem, $\boldsymbol{\theta}(z)$ is constant across the whole complex plane. Its constant value, denoted by $\boldsymbol{\theta}^{\infty}$, cannot be assumed to be null due to the existence of stresses at infinity. Consequently, a direct calculation gives the following expressions,

$$
\begin{aligned}
& \boldsymbol{B}_{+} \boldsymbol{f}_{+}^{\prime \prime}(z)=\overline{\boldsymbol{B}_{-}} \overline{\boldsymbol{f}_{-}^{\prime \prime}}(z), z \in S_{+} \\
& \boldsymbol{B}_{-} \boldsymbol{f}_{-}^{\prime \prime}(z)=\overline{\boldsymbol{B}_{+}} \overline{\boldsymbol{f}_{+}^{\prime \prime}}(z), z \in S_{-} .
\end{aligned}
$$

Displacement jump condition: Similarly, considering the displacement jump $\llbracket \mathbf{u} \rrbracket\left(x_{1}\right)=\mathbf{u}\left(x_{1}, 0^{+}\right)-\mathbf{u}\left(x_{1}, 0^{-}\right)$across the interface, and making use of the Stroh formalism in Eq. (14) yields,

$$
\llbracket \mathbf{u} \rrbracket\left(x_{1}\right)=\left[\boldsymbol{A}_{+} \boldsymbol{f}_{+}\left(x_{1}\right)+\overline{\boldsymbol{A}_{+} \boldsymbol{f}_{+}}\left(x_{1}\right)\right]-\left[\boldsymbol{A}_{-} \boldsymbol{f}_{-}\left(x_{1}\right)+\overline{\boldsymbol{A}_{-} \boldsymbol{f}_{-}}\left(x_{1}\right)\right] .
$$

Through mathematical manipulations and the analytic continuity argument defined in Eqs. (45) and (46), Eq. (47) yields,

$$
\frac{\partial^{2} \llbracket \mathbf{u} \rrbracket}{\partial x_{1}^{2}}\left(x_{1}\right)=-\mathrm{i}\left[\boldsymbol{H} \boldsymbol{B}_{+} \boldsymbol{f}_{+}^{\prime \prime}\left(x_{1}\right)-\overline{\boldsymbol{H}} \boldsymbol{B}_{-} \boldsymbol{f}_{-}^{\prime \prime}\left(x_{1}\right)\right]
$$

where the second-order bimaterial elastic constants tensor $\boldsymbol{H}$ is defined as $\boldsymbol{H}=$ $\boldsymbol{M}_{+}+\overline{\boldsymbol{M}_{-}}$with $\boldsymbol{M}_{m}=\mathrm{i} \boldsymbol{A}_{m} \boldsymbol{B}_{m}^{-1}, m=\{+,-\}$. As proven by Stroh (1958), the tensors $\boldsymbol{M}_{+}, \boldsymbol{M}_{-}$and $\boldsymbol{H}$ are all Hermitian and positive-definite matrices. Those properties are important for the existence of solutions to the problem.

Equation (48) can be rewritten to provide an equivalent boundary condition for the displacement jump across the interface directly related to the interface elastic properties, if we consider both the interfacial elasticity boundary conditions across the interface in Eq. (26) and the interfacial constitutive relationship in Eq.(25), such that,

$$
\begin{aligned}
\boldsymbol{H} \boldsymbol{B}_{+} \boldsymbol{f}_{+}^{\prime \prime}\left(x_{1}\right)-\overline{\boldsymbol{H}} \boldsymbol{B}_{-} \boldsymbol{f}_{-}^{\prime \prime}\left(x_{1}\right) & =\mathrm{i} \boldsymbol{\Lambda}^{\perp}\left[\boldsymbol{B}_{+} \boldsymbol{f}_{+}^{\prime \prime \prime}\left(x_{1}\right)+\overline{\boldsymbol{B}_{+}} \overline{\boldsymbol{f}_{+}^{\prime \prime \prime}}\left(x_{1}\right)\right] \\
& -\mathrm{i} \mathbb{H}_{I I}^{S}\left[\boldsymbol{A}_{+} \boldsymbol{f}_{+}^{\prime \prime \prime}\left(x_{1}\right)+\overline{\boldsymbol{A}}_{+} \overline{\boldsymbol{f}_{+}^{\prime \prime \prime}}\left(x_{1}\right)\right] .
\end{aligned}
$$


The real matrix $\mathbb{H}_{I I}^{S}$ is defined from the third-order tensor $\mathbb{H}^{S}$ as,

$$
\mathbb{H}_{I I i j}^{S}=\mathbb{H}_{i 1 j}^{S} \delta_{1 j}+\frac{1}{2}\left(\mathbb{H}_{i 13}^{S}+\mathbb{H}_{i 31}^{S}\right) \delta_{3 j}
$$

It should be noted that $\mathbb{H}_{I I}^{S}$ is singular while the second-order tensor $\boldsymbol{\Lambda}^{\perp}$, corresponding to the interface transverse compliance tensor, is real and assumed to be symmetric. Consequently, the matrix i $\Lambda^{\perp}$ is skew-Hermitian and therefore diagonalizable.

Elastic fields: Using the analytic continuity argument in Eqs. (45) and (46), Eq. (49) can be rearranged in such a way that one can isolate terms defined in the upper and lower half-planes respectively such that,

$$
\begin{gathered}
\boldsymbol{H} \boldsymbol{B}_{+} \boldsymbol{f}_{+}^{\prime \prime}\left(x_{1}\right)+\left[\mathbb{H}_{I I}^{S} \boldsymbol{M}_{+}-\mathrm{i} \boldsymbol{\Lambda}^{\perp}\right] \boldsymbol{B}_{+} \boldsymbol{f}_{+}^{\prime \prime \prime}\left(x_{1}\right)= \\
\overline{\boldsymbol{H}} \boldsymbol{B}_{-} \boldsymbol{f}_{-}^{\prime \prime}\left(x_{1}\right)+\left[\mathbb{H}_{I I}^{S} \overline{\boldsymbol{M}_{+}}+\mathrm{i} \boldsymbol{\Lambda}^{\perp}\right] \boldsymbol{B}_{-} \boldsymbol{f}_{-}^{\prime \prime \prime}\left(x_{1}\right) .
\end{gathered}
$$

Following the same reasoning used in the definition of the potential $\boldsymbol{\theta}(z)$, one can defined a complex potential $\boldsymbol{\omega}(z)$ defined in the upper and lower half-planes,

$$
\boldsymbol{\omega}(z)= \begin{cases}\boldsymbol{H} \boldsymbol{B}_{+} \boldsymbol{f}_{+}^{\prime \prime}(z)+\left[\mathbb{H}_{I I}^{S} \boldsymbol{M}_{+}-\mathrm{i} \boldsymbol{\Lambda}^{\perp}\right] & \boldsymbol{B}_{+} \boldsymbol{f}_{+}^{\prime \prime \prime}(z), z \in S_{+} \\ \overline{\boldsymbol{H}} \boldsymbol{B}_{-} \boldsymbol{f}_{-}^{\prime \prime}(z)+\left[\mathbb{H}_{I I}^{S} \boldsymbol{M}_{+}+\mathrm{i} \boldsymbol{\Lambda}^{\perp}\right] \boldsymbol{B}_{-} \boldsymbol{f}_{-}^{\prime \prime \prime}(z), z \in S_{-}\end{cases}
$$

Invoking the Liouville's theorem, $\boldsymbol{\omega}(z)$ is constant in the whole complex plane and can be assumed to be equal to zero since the transverse stresses (which are proportional to $\boldsymbol{f}^{\prime}$ ) are constant at infinity.

Consequently, if we define $\boldsymbol{\zeta}_{m}(z)=\boldsymbol{B}_{m} \boldsymbol{f}_{m}^{\prime \prime}(z)$, two simple equivalent eigenvalue problems can be defined in the upper and lower half-planes,

$$
\boldsymbol{\zeta}_{m}^{\prime}(z)+\mathbb{M}_{m} \boldsymbol{\zeta}_{m}(z)=0
$$

with $\mathbb{M}_{+}=\left[\mathbb{H}_{I I}^{S} \boldsymbol{M}_{+}-\mathrm{i} \boldsymbol{\Lambda}^{\perp}\right]^{-1} \boldsymbol{H}$ and $\mathbb{M}_{-}=\left[\mathbb{H}_{I I}^{S} \overline{\boldsymbol{M}_{+}}+\mathrm{i} \boldsymbol{\Lambda}^{\perp}\right]^{-1} \overline{\boldsymbol{H}}=\overline{\mathbb{M}_{+}}$and $m=\{+,-\}$. The function $\boldsymbol{\zeta}$ can be interpreted as the variation of the stress field throughout the continuum. Solutions to the eigenvalue problems in Eq. (53) define the elastic fields in both half-spaces while accounting for the interface properties. Note that when the interfacial elasticity is neglected, the present formulation coincides with the classical formulation for a perfectly bonded interface (Suo, 1990).

Analytical solutions to Eq. (53) requires the second order tensor $\mathbb{M}_{m}$ to be at least triangularizable. This condition is satisfied as long as its components are not all real numbers. In the rest of this manuscript, $\mathbb{M}_{m}$ is assumed to be diagonalizable such that $\mathbb{M}_{m}=\boldsymbol{U}_{m} \boldsymbol{D}_{m} \boldsymbol{U}_{m}^{-1}$, with $\boldsymbol{D}_{m}=\left\langle\lambda_{*}\right\rangle$ with $\lambda_{*}$ being the three distinct 
eigenvalues of this matrix. This diagonalization is only valid if $\lambda_{*} \neq 0$. Note that since $\mathbb{M}_{+}$and $\mathbb{M}_{-}$are conjugate, their eigenroots are conjugate from one another. Furthermore, the eigenvalues $\lambda_{\alpha}$ are complex-valued quantities that depend on the coupling between the bulk elastic moduli and the interfacial elastic moduli. The subscript $m$ can be dropped for the rest of the analysis. The diagonalized form of Eq. (53) can then be written as,

$$
\Psi^{\prime}(z)+\left\langle\lambda_{*}\right\rangle \Psi(z)=0
$$

with $\boldsymbol{\Psi}(z)=\boldsymbol{U}_{m}^{-1} \boldsymbol{\zeta}(z)$. The general solution to Eq. (54) is simply given by,

$$
\boldsymbol{\Psi}(z)=\left\langle e^{-\lambda_{*} z}\right\rangle \boldsymbol{K}_{\Psi}
$$

where $\boldsymbol{K}_{\Psi}$ is an integration complex vector. From Eq. (55), the stress in both halfplanes can be deduced by integration,

$$
\boldsymbol{B} \boldsymbol{f}^{\prime}(z)=\boldsymbol{K}_{\sigma}+\boldsymbol{U}\left\langle\frac{-e^{-\lambda_{*} z}}{\lambda_{*}}\right\rangle \boldsymbol{K}_{\Psi}
$$

where $\boldsymbol{K}_{\sigma}$ is another integration complex vector. It is important to note that, in the above, the complex variable $z$ differs from the Stroh argument $z_{*}=x_{1}+p_{*} x_{2}$ away from the interface i.e., when $x_{2} \neq 0$. Thus, expressions for the stress fields in the upper and lower half planes read,

$$
\begin{gathered}
\boldsymbol{\sigma}_{+}^{\perp}=2 \mathfrak{R e}\left(\boldsymbol{K}_{\sigma_{+}}\right)-2 \mathfrak{R e}\left(\boldsymbol{U}\left\langle\frac{e^{-\lambda_{*} z_{*}}}{\lambda_{*}}\right\rangle \boldsymbol{K}_{\Psi}\right) \\
\boldsymbol{\sigma}_{-}^{\perp}=2 \mathfrak{R e}\left(\boldsymbol{K}_{\sigma_{-}}\right)-2 \mathfrak{R e}\left(\overline{\boldsymbol{U}}\left\langle\frac{e^{-\bar{\lambda}_{*} z_{*}}}{\bar{\lambda}_{*}}\right\rangle \overline{\boldsymbol{K}}_{\Psi}\right) .
\end{gathered}
$$

The boundary conditions at infinity $\lim _{z \rightarrow \pm \infty} \boldsymbol{\sigma}^{\perp}=\boldsymbol{\sigma}^{\perp, \infty}$ can now be used to obtain the value of the $\boldsymbol{K}_{\sigma}$ integration constants (Ting, 1986),

$$
2 \mathfrak{R e}\left(\boldsymbol{K}_{\boldsymbol{\sigma}}\right)=\boldsymbol{\sigma}^{\perp, \infty}=2 \mathfrak{R e}\left(\boldsymbol{B}\left[\boldsymbol{A}^{T} \boldsymbol{g}_{\sigma}+\boldsymbol{B}^{T} \boldsymbol{h}_{\epsilon}\right]\right) .
$$

Note that the stress fields in Eqs. (57) and (58) for this seemingly imperfect interface are composed of a far field component (first term in the equations) corresponding the applied loads at infinity and a perturbation field (second term in the equations) characterized by the elastic response of the interface. Again if the effects of interfacial elasticity are neglected, the classical condition of traction continuity is recovered. 
The displacement fields in the upper and lower half planes can be defined in a similar fashion by using Eq. (56) and the components of the displacement vector at infinity defined in Eq. (39) such that,

$$
\begin{aligned}
& \boldsymbol{u}_{+}=2 \mathfrak{R e}\left(\boldsymbol{A}_{+} \boldsymbol{B}_{+}^{-1} \boldsymbol{U}\left\langle\frac{e^{-\lambda_{*} z_{*}}}{\lambda_{*}^{2}}\right\rangle \boldsymbol{K}_{\Psi}+\boldsymbol{A}_{+}\left\langle z_{*_{+}}\right\rangle\left[\boldsymbol{A}_{+}^{T} \boldsymbol{g}_{\sigma}+\boldsymbol{B}_{+}^{T} \boldsymbol{h}_{\epsilon}\right]+\boldsymbol{K}_{u_{+}}\right), \\
& \boldsymbol{u}_{-}=2 \mathfrak{R e}\left(\boldsymbol{A}_{-} \boldsymbol{B}_{-}^{-1} \overline{\boldsymbol{U}}\left\langle\frac{e^{-\lambda_{*} z_{*}}}{\bar{\lambda}_{*}^{2}}\right\rangle \overline{\boldsymbol{K}}_{\Psi}+\boldsymbol{A}_{-}\left\langle z_{*_{-}}\right\rangle\left[\boldsymbol{A}_{-}^{T} \boldsymbol{g}_{\sigma}+\boldsymbol{B}_{-}^{T} \boldsymbol{h}_{\epsilon}\right]+\boldsymbol{K}_{u_{-}}\right) .
\end{aligned}
$$

The complex constant vectors $\boldsymbol{K}_{u}$ result from the integration.

Determination of integration constants: At this point, two boundary conditions are needed to solve for the remaining integration constants. These conditions are: (i) the compatibility of the in-plane strain along the interface $\left(\boldsymbol{\epsilon}_{+}^{S}=\boldsymbol{\epsilon}_{-}^{S}=\boldsymbol{\epsilon}^{S}\right)$, and (ii) the displacement jump conditions across the interface through Eq. (26).

The in-plane strain compatibility condition yields,

$$
\boldsymbol{P}^{S} \cdot \boldsymbol{u}_{+}^{\prime}\left(x_{1}, 0\right)=\boldsymbol{P}^{S} \cdot \boldsymbol{u}_{-}^{\prime}\left(x_{1}, 0\right) .
$$

Since both Eq. (62) and Eq. (26) are valid across the whole interface, derivations leading to the expressions of the integration constants can be simplified by choosing an arbitrary but convenient value of $x_{1}$, e.g. $x_{1}=0$. Thus, after some manipulations, Eq. (62) can be rewritten as,

$$
\mathfrak{R e}\left(\mathrm{i} \boldsymbol{H} \boldsymbol{U}\left\langle\frac{1}{\lambda_{*}}\right\rangle \boldsymbol{K}_{\Psi}-\mathrm{i}\left[\boldsymbol{M}_{+}-\boldsymbol{M}_{-}\right] \boldsymbol{K}_{\sigma}\right)=0 .
$$

Eq. (63) establishes equality between the real parts of two tensorial products that include the constants $\boldsymbol{K}_{\Psi}$ and $\boldsymbol{K}_{\sigma}$, respectively. As a result, the complex integration constant $\boldsymbol{K}_{\Psi}$ can be expressed as a function of the bulk and interfacial elastic moduli in the following manner,

$$
\boldsymbol{K}_{\Psi}=\left\langle\lambda_{*}\right\rangle \boldsymbol{U}^{-1} \boldsymbol{H}^{-1}\left[\boldsymbol{M}_{+}-\boldsymbol{M}_{-}\right] \boldsymbol{K}_{\sigma},
$$

A couple of remarks should be noted here. Given the expression of $\boldsymbol{K}_{\Psi}$ in Eq. (64), one can see that the perturbation field results from a coupling between the "classical" mismatch between the elastic constants across the interface $\left(\boldsymbol{H}^{-1}\left[\boldsymbol{M}_{+}-\boldsymbol{M}_{-}\right]\right)$and the interface elastic properties $\left(\left\langle\lambda_{*}\right\rangle\right)$. For special combinations of the elastic constants, these effects could be suppressed: $\boldsymbol{K}_{\Psi}=0$ when (i) materials "+" and "-" share both the same elastic properties and orientation, or (ii) no loading is applied at infinity, i.e., $\boldsymbol{K}_{\sigma}=0$. 
Similarly, the displacement jump boundary condition across the interface is true for any value of $x_{1}$. As a result, for $x_{1}=0$, Eq. (26) can simply be written as,

$$
\llbracket \mathbf{u} \rrbracket(0,0)=\boldsymbol{\Delta}^{\perp},
$$

with $\Delta^{\perp}$ the interfacial strain defined in Eq. (25). As shown by Eq. (25), the interfacial strain $\boldsymbol{\Delta}^{\perp}$ depends on both the transverse stress $\boldsymbol{\sigma}^{\perp}$ and in-plane strain $\boldsymbol{\epsilon}^{S}$, which are continuous across the whole interface. Consequently, the interfacial strain constitutive relation is independent of the choice of the material used to express the in-plane strain and the transverse stress. In order to be consistent with what has been done previously (see Eq. (49)), the transverse stress and in-plane strain associated with medium "+" are used to express the interfacial strain. After some manipulations, Eq. (65) can be expressed to obtain the constant vectors $\boldsymbol{K}_{u_{+}}$and $\boldsymbol{K}_{u_{-}}$such that,

$$
\boldsymbol{K}_{u_{+}}-\boldsymbol{K}_{u_{-}}=\frac{1}{2}\left[\boldsymbol{\Lambda}_{0}^{\perp}+\mathbb{K}^{S}: \boldsymbol{\epsilon}^{m, S}\right]+\mathrm{i} \boldsymbol{H} \mathbb{M}_{+}^{-1} \boldsymbol{K}_{\sigma} .
$$

It is interesting to note that, in the absence of loading, Eq. (66) reduces to,

$$
\left.\llbracket \mathbf{u} \rrbracket\right|_{\left[\boldsymbol{h}_{\epsilon}, \boldsymbol{g}_{\sigma}\right]=[0,0]}=2 \mathfrak{R e}\left(\boldsymbol{K}_{u_{+}}-\boldsymbol{K}_{u_{-}}\right)=\boldsymbol{\Lambda}_{0}^{\perp}+\mathbb{K}^{S}: \boldsymbol{\epsilon}^{m, S} .
$$

This expression is consistent with the definition and physical interpretation of the interfacial strain as being the intrinsic thickness of the interface in a Gibbsian sense.

All together, Eqs. (57)-(61), Eq. (64), and Eq. (66) completely define the elastic fields for a seemingly imperfectly bonded anisotropic bimaterials while accounting for the interface elasticity.

To close this sub-section, a remark on the resolution technique used is warranted. The methodology described above is similar to the one used by Sudak and Wang (2006) and is based on complex analysis. However, an alternative approach (Koguchi and Suzuki, 2014) based on Fourier transform could have been used instead to obtain similar results.

\subsection{Solution for a Griffith crack lying between two perfectly bonded anisotropic ma- terials}

The third problem which considers a Griffith crack of length $L=2 a$ lying between two perfectly bonded anisotropic materials has already been studied and solved in the literature (Bassani and Qu, 1989a,b; Qu and Bassani, 1989, 1993; Suo, 1990). The main hypothesis consists of assuming two perfectly-bonded half-spaces, except in the cracked area. Through use of the superposition principle, loading of the crack faces is 
prescribed by the self-equilibrated tractions $\boldsymbol{t}^{*}\left(x_{1}\right)$ for $x_{1} \in[-a, a]$ corresponding to the imperfect interface problem described in Section 3.3. Consequently, the traction $\boldsymbol{t}^{*}\left(x_{1}\right)$ prescribed on the crack faces is given by,

$$
\boldsymbol{t}^{*}\left(x_{1}\right)=\boldsymbol{\sigma}^{\perp, \infty}-2 \mathfrak{R e}\left(\boldsymbol{U}\left\langle e^{-\lambda_{*} x_{1}}\right\rangle \boldsymbol{U}^{-1} \boldsymbol{H}^{-1}\left[\boldsymbol{M}_{+}-\boldsymbol{M}_{-}\right] \boldsymbol{K}_{\boldsymbol{\sigma}}\right)
$$

and no loading is applied at infinity. In the present problem, $\boldsymbol{t}^{*}\left(x_{1}\right)$ depends not only on the far field loading $\boldsymbol{\sigma}^{\perp, \infty}$ but also on the coupling between the interfacial properties and the bimaterial properties (again it is assumed that $\lambda_{*} \neq 0$ and distinct roots). In this configuration, the traction continuity condition across both the cracked and uncracked interface yields,

$$
\begin{aligned}
\boldsymbol{B}_{+} \boldsymbol{f}_{+}^{\prime}(z) & =\overline{\boldsymbol{B}_{-}} \overline{\boldsymbol{f}_{-}^{\prime}}(z), z \in S_{+}, \\
\boldsymbol{B}_{-} \boldsymbol{f}_{-}^{\prime}(z) & =\overline{\boldsymbol{B}_{+}} \overline{\boldsymbol{f}_{+}^{\prime}}(z), z \in S_{-} .
\end{aligned}
$$

Similarly, in this configuration, the continuity of the displacement across the perfectly bonded interface implies that one can define a complex potential $\gamma(z)$ which is analytic in the whole plane except on the crack lines, $C$ with,

$$
\boldsymbol{\gamma}(z)=\boldsymbol{B}_{+} \boldsymbol{f}_{+}^{\prime}(z)=\boldsymbol{H}^{-1} \overline{\boldsymbol{H}} \boldsymbol{B}_{-} \boldsymbol{f}_{-}^{\prime}(z), z \notin C
$$

As such, both the traction and displacement boundary conditions can then be rewritten in terms of $\gamma(z)$ in the form an heterogeneous Hilbert problem (Suo, 1990),

$$
\begin{aligned}
\boldsymbol{\gamma}_{+}\left(x_{1}\right)+\overline{\boldsymbol{H}}^{-1} \boldsymbol{H} \boldsymbol{\gamma}_{-}\left(x_{1}\right) & =-\boldsymbol{t}^{*}\left(x_{1}\right), \\
\boldsymbol{H}\left[\boldsymbol{\gamma}_{+}\left(x_{1}\right)-\boldsymbol{\gamma}_{-}\left(x_{1}\right)\right] & =\mathrm{i} \frac{\partial \llbracket \mathbf{u} \rrbracket}{\partial x_{1}}\left(x_{1}\right)=0 .
\end{aligned}
$$

The associated homogeneous Hilbert problem, i.e.,

$$
\boldsymbol{\gamma}_{+}\left(x_{1}\right)+\overline{\boldsymbol{H}}^{-1} \boldsymbol{H} \boldsymbol{\gamma}_{-}\left(x_{1}\right)=0
$$

has a solution of the form $\gamma(z)=\boldsymbol{w} z^{-\frac{1}{2}+\mathrm{i} \varepsilon}$, where $\boldsymbol{w}$ is a constant vector, and $\varepsilon$ is a constant real scalar denoted as the oscillatory index (Suo, 1990). Substituting $\boldsymbol{\gamma}_{+}$ and $\gamma_{-}$in Eq.(74) leads to the following eigenvalue problem,

$$
\overline{\boldsymbol{H}} \boldsymbol{w}=e^{2 \pi \varepsilon} \boldsymbol{H} \boldsymbol{w} .
$$

The three eigenpairs, solutions to this eigenvalue problem, are $\left(e^{2 \pi \varepsilon}, \boldsymbol{w}\right),\left(e^{-2 \pi \varepsilon}, \overline{\boldsymbol{w}}\right)$ and $\left(1, \boldsymbol{w}_{3}\right)$, and $\boldsymbol{w}$ and $\boldsymbol{w}_{3}$ a complex and real vector respectively. It should be 
noted that the three eigenvalues are distinct if and only if $\boldsymbol{H} \neq \overline{\boldsymbol{H}}$. Using the fact that every complex-valued vector can be represented as a linear combination of the three eigenvectors defined above, the final solution of the heterogeneous Hilbert problem (Suo, 1990) is given as,

$$
\boldsymbol{\gamma}(z)=\gamma_{1}(z) \boldsymbol{w}+\gamma_{2}(z) \overline{\boldsymbol{w}}+\gamma_{3}(z) \boldsymbol{w}_{3}
$$

where,

$$
\begin{aligned}
& \gamma_{1}(z)=\frac{\chi(z)}{2 \pi \mathrm{i}} \int_{C} \frac{t_{1}^{*}(\xi)}{\chi_{+}(\xi)(\xi-z)} d \xi \\
& \gamma_{2}(z)=\frac{\bar{\chi}(z)}{2 \pi \mathrm{i}} \int_{C} \frac{\bar{t}_{1}^{*}(\xi)}{\bar{\chi}_{+}(\xi)(\xi-z)} d \xi \\
& \gamma_{3}(z)=\frac{\chi_{0}(z)}{2 \pi \mathrm{i}} \int_{C} \frac{t_{3}^{*}(\xi)}{\chi_{+}^{0}(\xi)(\xi-z)} d \xi
\end{aligned}
$$

and the complex functions $\chi(z)$ and $\chi^{0}(z)$ are defined as,

$$
\begin{aligned}
\chi^{0}(z) & =\left(z^{2}-a^{2}\right)^{-\frac{1}{2}} \\
\chi(z) & =\chi^{0}(z)\left(\frac{z-a}{z+a}\right)^{\mathrm{i} \varepsilon}
\end{aligned}
$$

The tractions $t_{1}^{*}(\xi), \bar{t}_{1}^{*}(\xi)$ and $t_{3}^{*}(\xi)$ are defined by the inner product of Eq. (72) with $\overline{\boldsymbol{w}}^{T} \boldsymbol{H}, \boldsymbol{w}^{T} \boldsymbol{H}$, and $\boldsymbol{w}_{3}^{T} \boldsymbol{H}$ such that (Suo, 1990),

$$
t_{1}^{*}=-\frac{\overline{\boldsymbol{w}}^{T} \boldsymbol{H} \boldsymbol{t}^{*}}{\overline{\boldsymbol{w}}^{T} \boldsymbol{H} \boldsymbol{w}}, \bar{t}_{1}^{*}=-\frac{\boldsymbol{w}^{T} \boldsymbol{H} \boldsymbol{t}^{*}}{\boldsymbol{w}^{T} \boldsymbol{H} \overline{\boldsymbol{w}}}, t_{3}^{*}=-\frac{\boldsymbol{w}_{3}^{T} \boldsymbol{H} \boldsymbol{t}^{*}}{\boldsymbol{w}_{3}{ }^{T} \boldsymbol{H} \boldsymbol{w}_{3}} .
$$

Once $\gamma(z)$ is obtained, the full-field solution in both materials $S_{+}$and $S_{-}$can be defined by Eq. (71) along the $\boldsymbol{w}_{3}$ and in the plane spanned by $\mathfrak{R e}(\boldsymbol{w})$ and $\mathfrak{I m}(\boldsymbol{w})$ (Suo, 1990).

\section{Nature of crack tip singularity and oscillatory effects}

In the previous sections, the elastic fields about a crack tip in an anisotropic bimaterial have been studied by integral equations and complex variable approaches. In order to clarify the singular behavior near the crack tip, the complex potential $\gamma(z)$ and traction along the interface are briefly reconsidered hereafter.

The scalar tractions $t_{1}^{*}\left(x_{1}\right), \bar{t}_{1}^{*}\left(x_{1}\right)$ and $t_{3}^{*}\left(x_{1}\right)$ obtained from the self-equilibrated traction vector $\boldsymbol{t}^{*}\left(x_{1}\right)$ of the imperfect interface problem can be decomposed into 
the sum of a constant and spatially varying terms. As a result, the complex functions $\gamma_{i}(z), i=\{1,2,3\}$, solutions to the heterogeneous Hilbert problem defined in Eqs. (72)-(73), can be expressed as,

$$
\begin{aligned}
\boldsymbol{t}^{*}\left(x_{1}\right) & =\boldsymbol{t}_{0}^{*}-\boldsymbol{\delta} \mathbf{t}^{*}\left(x_{1}\right)=\boldsymbol{\sigma}^{\perp, \infty}-\boldsymbol{\delta} \mathbf{t}^{*}\left(x_{1}\right), \\
\gamma_{1}(z) & =\frac{\chi(z)}{2 \pi} \int_{-a}^{a} \frac{t_{01}^{*}-\delta t_{1}^{*}(\xi)}{\chi_{+}(\xi)(\xi-z)} d \xi=\frac{\chi(z)}{2 \pi} q_{1}^{*}(z), \\
\gamma_{2}(z) & =\frac{\bar{\chi}(z)}{2 \pi} \int_{-a}^{a} \frac{\bar{t}_{01}^{*}-\overline{\delta t_{1}^{*}(\xi)}}{\bar{\chi}_{+}(x)(\xi-z)} d \xi=\frac{\bar{\chi}(z)}{2 \pi} \bar{q}_{1}^{*}(z), \\
\gamma_{3}(z) & =\frac{\chi_{0}(z)}{2 \pi} \int_{-a}^{a} \frac{t_{03}^{*}-\delta t_{3}^{*}(\xi)}{\chi_{+}^{0}(\xi)(\xi-z)} d \xi=\frac{\chi_{0}(z)}{2 \pi} q_{3}^{*}(z),
\end{aligned}
$$

where the constant terms are equal to,

$$
t_{01}^{*}=-\frac{\overline{\boldsymbol{w}}^{T} \boldsymbol{H} \boldsymbol{\sigma}^{\perp, \infty}}{\overline{\boldsymbol{w}}^{T} \boldsymbol{H} \boldsymbol{w}}, t_{03}^{*}=-\frac{\boldsymbol{w}_{3}{ }^{T} \boldsymbol{H} \boldsymbol{\sigma}^{\perp, \infty}}{\boldsymbol{w}_{3}{ }^{T} \boldsymbol{H} \boldsymbol{w}_{3}},
$$

and the spatially varying terms are given by,

$$
\boldsymbol{\delta t}^{*}\left(x_{1}\right)=2 \mathfrak{R e}\left(\boldsymbol{U}\left\langle e^{-\mathfrak{R e}\left(\lambda_{*}\right) x_{1}-\mathrm{i} \mathfrak{I m}\left(\lambda_{*}\right) x_{1}}\right\rangle \boldsymbol{U}^{-1} \boldsymbol{H}^{-1}\left[\boldsymbol{M}_{+}-\boldsymbol{M}_{-}\right]\right) \boldsymbol{K}_{\sigma}
$$

with the projections $\delta t_{1}^{*}(\xi)$ and $\delta t_{3}^{*}(\xi)$ being defined by,

$$
\delta t_{1}^{*}(\xi)=-\frac{\overline{\boldsymbol{w}}^{T} \boldsymbol{H} \boldsymbol{\delta} \mathbf{t}^{*}(\xi)}{\overline{\boldsymbol{w}}^{T} \boldsymbol{H} \boldsymbol{w}}, \delta t_{3}^{*}(\xi)=-\frac{\boldsymbol{w}_{3}{ }^{T} \boldsymbol{H} \boldsymbol{\delta} \mathbf{t}^{*}(\xi)}{\boldsymbol{w}_{3}{ }^{T} \boldsymbol{H} \boldsymbol{w}_{3}}
$$

The scalar complex functions $q_{i}$ correspond to the projections along the vectors $\boldsymbol{w}, \overline{\boldsymbol{w}}$ and $\boldsymbol{w}_{3}$ of a generalized weighted loading vector. They are composed of two terms. A constant term arises from the uniform loading applied at infinity which corresponds to the classical oscillatory solution (Qu and Bassani, 1989; Qu and Li, 1991) with an oscillatory index $\varepsilon$. The perturbation terms correspond to the coupling between the interfacial elasticity constants and the bimaterial elastic constants.

The singular stress field along the interface (i.e. $x_{2}=0$ ) near the crack tip is obtained by considering the limit of the functions $\gamma_{i}$ as $x_{1} \rightarrow a^{+}$such that,

$$
\begin{aligned}
& \gamma_{1}\left(x_{1}\right) \propto \frac{\left(x_{1}-a\right)^{\mathrm{i} \varepsilon}}{\sqrt{x_{1}-a}} q_{1}^{*}(a) \\
& \gamma_{3}\left(x_{1}\right) \propto \frac{1}{\sqrt{x_{1}-a}} q_{3}^{*}(a)
\end{aligned}
$$


with

$$
\begin{aligned}
& q_{1}^{*}(a)=-\cosh \pi \varepsilon \int_{-a}^{a}\left(\frac{a+\xi}{a-\xi}\right)^{\frac{1}{2}+\mathrm{i} \varepsilon}\left[t_{01}^{*}-\delta t_{1}^{*}(\xi)\right] d \xi \\
& q_{3}^{*}(a)=-\int_{-a}^{a}\left(\frac{a+\xi}{a-\xi}\right)^{\frac{1}{2}}\left[t_{03}^{*}-\delta t_{3}^{*}(\xi)\right] d \xi .
\end{aligned}
$$

It is interesting to note from Eqs. (90)-(91) that considerations of the interfacial elasticity leave the order of the singularity untouched but affect the oscillatory effects as also observed recently by Sigaeva and Schiavone (2016) for isotropic bimaterials. More specifically, by normalizing over the crack half-length, we can assume that the perturbation terms associated with the $\gamma_{i}$ potentials along the interface $\left(z=x_{1}\right.$, and $x_{1}$ corresponds to the distance from the crack tip) are proportional to a complex function of type $f\left(x_{1}\right)$ such that,

$$
f\left(x_{1}\right)=\int_{-1}^{1} \frac{\sqrt{1-\xi^{2}}}{\xi-x_{1}}\left(\frac{1+\xi}{1-\xi}\right)^{\mathrm{i} \varepsilon} e^{-\mathfrak{R e}(\lambda) \xi} e^{-\mathrm{i} \mathfrak{I m}(\lambda) \xi} d \xi
$$

Using the same normalization with respect to the half-crack length $a$ utilsed for the function $f$ in Eq. (94), the perturbation component $\delta \gamma$ of the complex potential $\gamma_{1}$ accounting for the interface mechanical behavior can be represented and decomposed as follows,

$$
\begin{aligned}
\delta \gamma & =\frac{\chi\left(x_{1}\right)}{2 \pi} \int_{-1}^{1} \frac{\sqrt{1-\xi^{2}}}{\xi-x_{1}}\left(\frac{1+\xi}{1-\xi}\right)^{\mathrm{i} \epsilon} \delta t_{1}^{*}(\xi) d \xi \\
& =\frac{\chi\left(x_{1}\right)}{2 \pi} \sum_{i=1}^{3} \mu_{i} \int_{-1}^{1} \frac{\sqrt{1-\xi^{2}}}{\xi-x_{1}}\left(\frac{1+\xi}{1-\xi}\right)^{\mathrm{i} \epsilon} e^{-\mathfrak{R e}\left(\lambda_{i}\right) \xi} e^{-\mathrm{i} \mathfrak{I m}\left(\lambda_{i}\right) \xi} d \xi \\
& =\frac{\chi_{0}\left(x_{1}\right)}{2 \pi} \cos \left[\epsilon \ln \left(\frac{x_{1}-1}{x_{1}+1}\right)\right] \sum_{i=1}^{3}\left[\alpha_{i} \mathfrak{R e} f_{i}\left(x_{1}\right)-\beta_{i} \mathfrak{I m} f_{i}\left(x_{1}\right)\right] \\
& -\frac{\chi_{0}\left(x_{1}\right)}{2 \pi} \sin \left[\epsilon \ln \left(\frac{x_{1}-1}{x_{1}+1}\right)\right] \sum_{i=1}^{3}\left[\beta_{i} \mathfrak{R e} f_{i}\left(x_{1}\right)+\alpha_{i} \mathfrak{I m} f_{i}\left(x_{1}\right)\right] \\
& +\mathrm{i} \frac{\chi_{0}(x)}{2 \pi} \cos \left[\epsilon \ln \left(\frac{x_{1}-1}{x_{1}+1}\right)\right] \sum_{i=1}^{3}\left[\beta_{i} \mathfrak{R e} f_{i}\left(x_{1}\right)+\alpha_{i} \mathfrak{I m} f_{i}\left(x_{1}\right)\right] \\
& +\mathrm{i} \frac{\chi_{0}(x)}{2 \pi} \sin \left[\epsilon \ln \left(\frac{x_{1}-1}{x_{1}+1}\right)\right] \sum_{i=1}^{3}\left[\alpha_{i} \mathfrak{R e} f_{i}\left(x_{1}\right)-\beta_{i} \mathfrak{I m} f_{i}\left(x_{1}\right)\right]
\end{aligned}
$$


where the functions $f_{i}$ correspond to the function $f$ associated with the eigenvalue $\lambda_{i}$, and $\alpha_{i}$ and $\beta_{i}$ denote the real and imaginary parts of the complex scalar $\mu_{i}$, respectively, with $i=\{1,2,3\}$. Clearly, from Eq. (95), both $\mathfrak{R e}(f)$ and $\mathfrak{I m}(f)$ have an impact on the oscillatory behavior of the interface-dependent component of $\gamma_{1}$ in terms of its amplitude and phase. In other words, considerations of the interfacial elasticity through $f\left(x_{1}\right)$ clearly affects the oscillatory behavior in the vicinity of the crack tip via the generalized loading vector. Depending on the values associated with both the real and imaginary part of the eigenvalues $\lambda$ (which are associated with the surface elasticity constants), constructive/destructive "interferences" in the vicinity of the crack tip can occur in conjunction with the classical oscillatory behavior. This point is illustrated in the parametric study in Fig. 3 on the modulus and argument of the function $f$, for a typical oscillatory index $\varepsilon=0.04$ (Suo, 1990). As shown in Fig. 3 (a) and (c), the modulus of the complex function $f$ shows a strong dampening or amplification dependence on both the real and imaginary parts of $\lambda$. Similarly, the variation of the argument of the complex function $f$ shown in Fig. 3 (b) and (d) highlights the significant impact of the imaginary part of $\lambda$ on the interference effect with the oscillatory behavior, while the real part of $\lambda$ seems to induce a phase change. As a corollary to this parametric study, it appears that the magnitude and the argument of the generalized loading vector are primarily controlled by the real and imaginary parts of the eigenvalues associated with the newly introduced coupling tensor $\mathbb{M}$, respectively. Values of the surface elasticity constants are not only dependent on the bimaterial system of interest (grain boundaries vs. phase boundaries), but also on interface structure in terms of the coherency and plane orientation. Detailed atomistic-type simulations (Dingreville and $\mathrm{Qu}, 2009$ ) are required to calculate and quantify such properties.

Analogous to the definition provided by Suo (1990) and Qu and Li (1991), the stress intensity factor $\boldsymbol{k}=\left[k_{I}, k_{I I}, k_{I I I}\right]^{T}$ is readily obtained from Eqs. (84)-(86) such that,

$$
\begin{aligned}
k_{I} & =\lim _{x_{1} \rightarrow a^{+}} 2 \sqrt{2 \pi} \sqrt{x_{1}-a}\left(x_{1}-a\right)^{-\mathrm{i} \varepsilon} \gamma_{1}\left(x_{1}\right), \\
k_{I I I} & =\lim _{x_{1} \rightarrow a^{+}} 2 \sqrt{2 \pi} \sqrt{x_{1}-a} \gamma_{3}\left(x_{1}\right)
\end{aligned}
$$



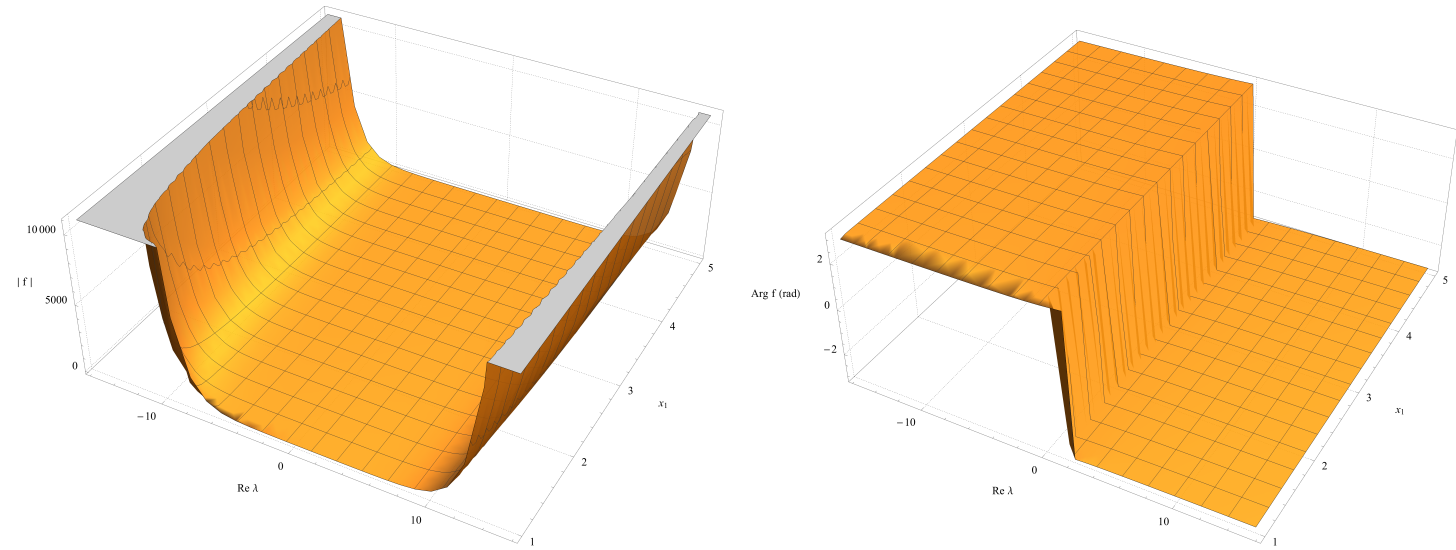

(a) $\left.|f|\right|_{\mathfrak{R e}(\lambda) \neq 0 ; \Im \mathfrak{m}(\lambda)=0}$

(b) $\left.\operatorname{Arg}(f)\right|_{\mathfrak{R e}(\lambda) \neq 0 ; \mathfrak{I m}(\lambda)=0}$
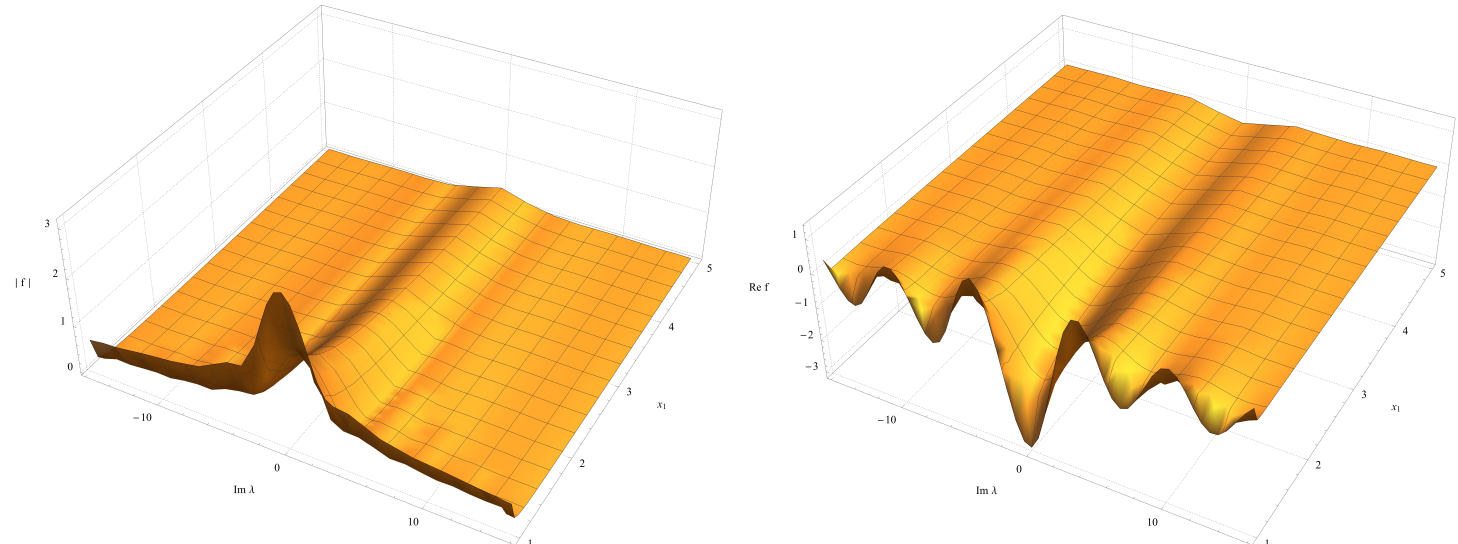

(c) $\left.|f|\right|_{\mathfrak{R e}(\lambda)=0 ; \Im \mathfrak{m}(\lambda) \neq 0}$

(d) $\left.\operatorname{Arg}(f)\right|_{\mathfrak{R e}(\lambda)=0 ; \mathfrak{I m}(\lambda) \neq 0}$

Figure 3: Parametric analysis of the amplitude and frequency response of the function $f\left(x_{1}\right)$ defined in Eq.(94) when $\mathfrak{R e}(\lambda) \in[-4 \pi, 4 \pi]$ and $\mathfrak{I m}(\lambda) \in[-4 \pi, 4 \pi]$. The oscillatory index $\varepsilon$ is taken as $\varepsilon=-0.04$.

or equivalently,

$$
\begin{aligned}
k_{I} & =-\sqrt{\frac{2}{\pi}} \cosh \pi \varepsilon\left(\frac{1}{2 a}\right)^{\frac{1}{2}+\mathrm{i} \varepsilon} \int_{-a}^{a}\left(\frac{a+\xi}{a-\xi}\right)^{\frac{1}{2}+\mathrm{i} \varepsilon}\left[t_{01}^{*}-\delta t_{1}^{*}(\xi)\right] d \xi, \\
& =\sqrt{\frac{2}{\pi}}\left(\frac{1}{2 a}\right)^{\frac{1}{2}+\mathrm{i} \varepsilon} q_{1}^{*}(a), \\
k_{I I I} & =-\sqrt{\frac{1}{\pi a}} \int_{-a}^{a}\left(\frac{a+\xi}{a-\xi}\right)^{\frac{1}{2}}\left[t_{03}^{*}-\delta t_{3}^{*}(\xi)\right] d \xi \\
& =\sqrt{\frac{1}{\pi a}} q_{3}^{*}(a) .
\end{aligned}
$$


where the three fracture modes are characterized with respect to the projections along the vectors $\boldsymbol{w}, \overline{\boldsymbol{w}}$ and $\boldsymbol{w}_{3}$ of a generalized weighted loading vector. The cracktip stress field have the standard square root singularity, but while the fracture modes are classically decoupled in terms of the load vector, the stress intensity factors do depend on the coupling of the bimaterial and the interface materials constants.

\section{Conclusion}

The basic notions of interfacial fracture mechanics are extended to include interfacial effects such as the structural mismatch and the associated interfacial elastic response. By adopting the concept of interfacial elasticity (in the Gibbsian sense), the boundary conditions introduced are equivalent to those for a weakly bounded interfaces, i.e. the displacement jump across the interface is proportional to the traction component in terms of interfacial elastic constants. Resolution of this problem is carried out by decomposing it into three simpler problems consisting of (i) the problem considering an uncracked bimaterial of two perfectly bonded anisotropic materials subjected to the eigenstrain, (ii) the problem of a seemingly imperfectly bonded bimaterial and subjected to known stresses at infinity and (iii) the problem of a bimaterial containing a crack lying along a perfectly bonded interface with traction forces applied on the crack faces.

One important result stemming from this formulation is that the perturbation of the elastic fields in the vicinity of the crack tip can not only be directly related to the classical difference between the elastic constants of both anisotropic solids but also to the elastic properties of the interface itself and its coupling with the bicrystal elastic constants. While the consideration of the interfacial elasticity does not affect the order of the singularity, it modifies the oscillatory effects associated with problems involving interface cracks by providing a constructive/destructive "interference" effect. This general formulation provides an insight on the physical significance and the obvious coupling between the interface structure and the associated mechanical fields in the vicinity of the crack tip. The incorporation of such interfacial properties provides a direct link between lower scale descriptions (Dingreville and Qu, 2009; Barrows et al., 2016) of the near-tip behavior, e.g. atomistic simulations, and their continuum description as described in this work.

\section{Acknowledgment}

Supported by the Laboratory Directed Research and Development program at Sandia National Laboratories, a multi-mission laboratory managed and operated by Sandia Corporation, a wholly owned subsidiary of Lockheed Martin Corporation, for 
the U.S. Department of Energy's National Nuclear Security Administration under contract DE-AC04-94AL85000. The authors thank Scott Grutzik, Jianmin Qu and Stéphane Berbenni for their comments and suggestions on this manuscript. 


\section{References}

Antipov, Y. A., Avila-Pozos, O., Kolaczkowski, S. T., Movchan, A. B., 2001. Mathematical model of delamination cracks on imperfect interfaces. International Journal of Solids and Structures 38 (36), 6665-6697.

Antipov, Y. A., Schiavone, P., 2011. Integro-differential equation for a finite crack in a strip with surface effects. The Quarterly Journal of Mechanics and Applied Mathematics 64 (1), 87-106.

Barrows, W., Dingreville, R., Spearot, D., 2016. Traction-separation relationships for hydrogen induced grain boundary embrittlement in nickel via molecular dynamics simulations. Materials Science and Engineering: A 650, 354-364.

Bassani, J. L., Qu, J., 1989a. Finite crack on bimaterial and bicrystal interfaces. Journal of the Mechanics and Physics of Solids 37 (4), 435-453.

Bassani, J. L., Qu, J., 1989b. On elasticity solutions for cracks on bimaterial and bicrystal interfaces. Materials Science and Engineering, A 107, 177-184.

Cahn, J. W., Larché, F., 1982. Surface stress and the chemical equilibrium of small crystals-II. Solid particles embedded in a solid matrix. Acta Metallurgica 30 (1), $51-56$.

Chadwick, P., Smith, G. D., 1977. Foundations of the theory of surface waves in anisotropic elastic materials. Advances in Applied Mechanics 17 (C), 303-376.

Cheng, Z.-Q., Jemah, A. K., Williams, F. W., 1996. Theory for multilayered anisotropic plates with weakened interfaces. Journal of Applied Mechanics 63 (4), $1019-1026$.

Clements, D. L., 1971. A crack between dissimilar anisotropic media. International Journal of Engineering Science 9 (2), 257-265.

Comninou, M., 1977. The interface crack. Journal of Applied Mechanics 44 (4), 631636.

Comninou, M., 1990. An overview of interface cracks. Engineering Fracture Mechanics 37 (1), 197-208.

Deng, X., 1993. General crack-tip fields for stationary and steadily growing interface cracks in anisotropic bimaterials. Journal of Applied Mechanics 60 (1), 183-189. 
Dingreville, R., 2007. Modeling and characterization of the elastic behavior of interfaces in nanostructured materials: from an atomistic description to a continuum approach. Ph.D. thesis, Georgia Institute of Technology.

Dingreville, R., Hallil, A., Berbenni, S., 2014. From coherent to incoherent mismatched interfaces: A generalized continuum formulation of surface stresses. Journal of the Mechanics and Physics of Solids 72, 40-60.

Dingreville, R., Qu, J., 2009. A semi-analytical method to estimate interface elastic properties. Computational Materials Science 46 (1), 83-91.

Dundurs, J., 1969. Discussion: "Edge-bonded dissimilar orthogonal elastic wedges under normal and shear loading" (Bogy, DB, 1968, ASME J. Appl. Mech., 35, pp. 460-466). Journal of Applied Mechanics 36 (3), 650-652.

England, A. H., 1965. A crack between dissimilar media. Journal of Applied Mechanics 32 (2), 400-402.

England, A. H., 2003. Complex Variable Methods in Elasticity. Dover Publications, inc.

Eshelby, J. D., Read, W. T., Shockley, W., 1953. Anisotropic elasticity with applications to dislocation theory. Acta Metallurgica 1 (3), 251-259.

Feng, X.-Q., Wang, T.-J., Gao, W., 2008. Surface effects on the near-tip stresses for mode-I and mode-III cracks. Journal of Applied Mechanics 75, 011001.

Gao, H., 1991. Weight function analysis of interface cracks: Mismatch versus oscillation. Journal of Applied Mechanics 58 (4), 931-938.

Gao, H., Abbudi, M., Barnett, D. M., 1992. Interfacial crack-tip field in anisotropic elastic solids. Journal of the Mechanics and Physics of Solids 40 (2), 393-416.

Gautesen, A. K., Dundurs, J., 1988. The interface crack under combined loading. Journal of Applied Mechanics 55 (3), 580-586.

Gibbs, J. W., 1928. The collected works of J. Willard Gibbs, Volume I: Thermodynamics. Longmans, Green and Company, New York, 228.

Gotoh, M., 1967. Some problems of bonded anisotropic plates with cracks along the bond. International Journal of Fracture Mechanics 3 (4), 253-265. 
Gurtin, M. E., Murdoch, A. I., 1975. A continuum theory of elastic material surfaces. Archive for Rational Mechanics and Analysis 57 (4), 291-323.

Hwu, C., 2010. Anisotropic elastic plates. Springer Science \& Business Media.

Inglish, C. E., 1913. Stresses in a plate due to the presence of cracks and sharp corners. Transactions of the Institute of Naval Architects 60, 219-230.

Irwin, G. R., 1957. Analysis of stresses and strains near the end of a crack traversing a plate. Journal of Applied Mechanics 24, 361-364.

Kim, C. I., Schiavone, P., Ru, C.-Q., 2011. Effect of surface elasticity on an interface crack in plane deformations. Proceedings of the Royal Society of London A: Mathematical, Physical and Engineering Sciences 467, 3530-3549.

Koguchi, H., Hirasawa, Y., 2015. Stress and displacement fields around misfit dislocation in anisotropic dissimilar materials with interface stress and interface elasticity. Journal of Applied Mechanics 82 (8), 081005.

Koguchi, H., Suzuki, N., 2014. Singular stress fields in anisotropic bonded joints considering interface stress and interface elasticity. Journal of Applied Mechanics $81(7), 071003$.

Lekhnitskii, S. G., 1963. Theory of elasticity of an anisotropic body. San Francisco: Holden-Day.

Mishuris, G. S., Kuhn, G., 2001. Asymptotic behaviour of the elastic solution near the tip of a crack situated at a nonideal interface. ZAMM-Journal of Applied Mathematics and Mechanics/Zeitschrift für Angewandte Mathematik und Mechanik 81 (12), 811-826.

Nan, H., Wang, B., 2012. Effect of residual surface stress on the fracture of nanoscale materials. Mechanics Research Communications 44, 30-34.

Ni, L., Nemat-Nasser, S., 1991. Interface cracks in anisotropic dissimilar materials: an analytic solution. Journal of the Mechanics and Physics of Solids 29 (1), 113 144 .

Pan, E., 2003. Three-dimensional Green's functions in anisotropic elastic bimaterials with imperfect interfaces. Journal of Applied Mechanics 70 (2), 180-190.

Qu, J., Bassani, J. L., 1989. Interfacial fracture mechanics for anisotropic bimaterials. Journal of Mechanics and Physics of Solids 37 (4), 417-433. 
Qu, J., Bassani, J. L., 1993. Interfacial fracture mechanics for anisotropic bimaterials. Journal of Applied Mechanics 60 (2), 422-431.

Qu, J., Li, Q., 1991. Interfacial dislocation and its applications to interface cracks in anisotropic bimaterials. Journal of Elasticity 26 (2), 169-195.

Rice, J. R., 1988. Elastic fracture mechanics concepts for interfacial cracks. Journal of Applied Mechanics 55 (1), 98-103.

Romanov, A. E., Wagner, T., 2001. On the universal misfit parameter at mismatched interfaces. Scripta Materialia 45 (3), 325-331.

Romanov, A. E., Wagner, T., Rühle, M., 1998. Coherent to incoherent transition in mismatched interfaces. Scripta Materialia 38 (6), 869-875.

Shih, C. F., Asaro, R. J., 1990. Elastic-plastic and asymptotic fields of interface cracks. International Journal of Fracture 42 (2), 101-116.

Sigaeva, T., Schiavone, P., 2016. The effect of surface stress on an interface crack in linearly elastic materials. Mathematics and Mechanics of Solids 21 (6), 649-656.

Stroh, A. N., 1958. Dislocations and cracks in anisotropic elasticity. Philosophical Magazine 3, 625-649.

Stroh, A. N., 1962. Steady state problems in anisotropic elasticity. Journal of Mathematics and Physics 41 (1), 77-103.

Sudak, L. J., 2003. On the interaction between a dislocation and a circular inhomogeneity with imperfect interface in antiplane shear. Mechanics Research Communications 30 (1), 53-59.

Sudak, L. J., Wang, X., 2006. Green's function in plane anisotropic bimaterials with imperfect interface. IMA Journal of Applied Mathematics 71 (5), 783-794.

Suo, Z., 1990. Singularities, interfaces and cracks in dissimilar anisotropic media. Proceedings of the Royal Society of London. Series A, Mathematical and Physical 427 (1873), 331-358.

Suo, Z., Hutchinson, J. W., 1990. Interface crack between two elastic layers. International Journal of Fracture 43 (1), 1-18. 
Ting, T. C. T., 1986. Explicit solution and invariance of the singularities at an interface in anisotropic media. International Journal of Solids and Structures 22 (9), 965-983.

Ting, T. C. T., 1996. Existence of an extraordinary degenerate matrix $\mathrm{N}$ for anisotropic elastic materials. The Quarterly Journal of Mechanics and Applied Mathematics 49 (3), 405-417.

Ting, T. C. T., 2000. Recent developments in anisotropic elasticity. International Journal of Solids and Structures 37 (1), 401-409.

Ting, T. C. T., Hwu, C., 1988. Sextic formalism in anisotropic elasticity for almost non-semisimple matrix N. International Journal of Solids and Structures 24 (1), $65-76$.

Vellender, A., Pryce, L., Zagnetko, A., 2016. Integral identities for fracture along imperfectly joined anisotropic ceramic bimaterials. Journal of the European Ceramic Society 36 (9), 2389-2402.

Wang, X., Zhou, K., Wu, M. S., 2015. Interface cracks with surface elasticity in anisotropic bimaterials. International Journal of Solids and Structures 59, 110 120.

Williams, M. L., 1959. The stresses around a fault or crack in dissimilar media. Bulletin of the Seismology Society of America 49 (2), 199-204.

Willis, J. R., 1971. Fracture mechanics of interfacial cracks. Journal of the Mechanics and Physics of Solids 19 (6), 353-368.

Wu, K.-C., 1990. Stress intensity factor and energy release rate for interfacial cracks between dissimilar anisotropic materials. Journal of Applied Mechanics 57 (4), $882-886$. 


\section{Appendix A. Nomenclature and list of symbols}

We denote in boldface a vector a and matrix $\mathbf{A}$ of the Euclidian space $\mathbb{E}$ only by its element $a_{i}$ (i.e. $\left.\mathbf{a}=a_{i} \hat{\mathbf{e}}_{i}\right), A_{i j}\left(\mathbf{A}=A_{i j} \hat{\mathbf{e}}_{i} \otimes \hat{\mathbf{e}}_{j}\right)$, where the Einstein's convention of summation over repeated indices is used. Roman indices used in tensorial notations range from 1 to 3 (three-dimensional) and Greek indices range from 1 to 2 (two-dimensional), unless otherwise indicated.

\section{Notation indexing:}

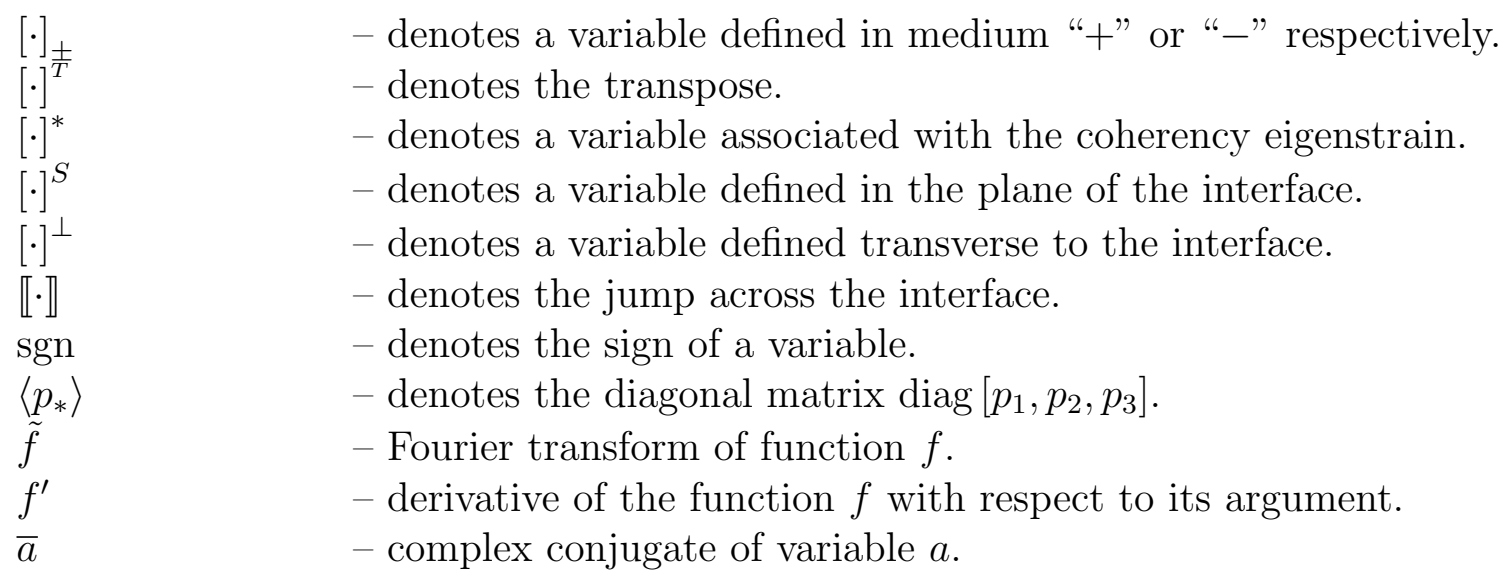

\section{Strain and stress definitions:}

$\begin{array}{ll}\mathbf{u} & \text { - displacement field. } \\ \boldsymbol{\epsilon} & - \text { strain tensor. } \\ \boldsymbol{\sigma} & - \text { stress tensor. } \\ \boldsymbol{\epsilon}^{S} & - \text { interfacial in-plane strain tensor. } \\ \boldsymbol{\sigma}^{\perp} & - \text { transverse stress tensor. } \\ \boldsymbol{\epsilon}^{*, S} & - \text { coherency eigenstrain tensor. } \\ \boldsymbol{\epsilon}^{0, S} & - \text { in-plane molar volume change eigenstrain tensor. } \\ \boldsymbol{\epsilon}^{m, S} & - \text { in-plane structural mismatch strain tensor. } \\ g(\mathbf{x}) & - \text { spatial variation of the structural mismatch. } \\ \varphi(\mathbf{x}) & - \text { variation of function } g(\mathbf{x}) \text {. } \\ \boldsymbol{\epsilon}^{*} & - \text { eigenstrain tensor. } \\ \varpi^{*} & - \text { virtual coherency body forces vector. } \\ \boldsymbol{\Phi} & - \text { stress function. } \\ \boldsymbol{t}^{*} & - \text { traction distribution across crack segment. }\end{array}$




\section{Bulk constants:}
$\mathbb{C}$
- stiffness elasticity tensor.
$Q, T, R$
- subsets of stiffness tensor $\mathbb{C}$.

\section{Interfacial constants:}

\begin{tabular}{|c|c|}
\hline$\Gamma$ & - interfacial excess energy. \\
\hline$\Gamma_{0}$ & - residual interfacial excess energy. \\
\hline$\Sigma^{S}$ & - coherent surface stress. \\
\hline$\Upsilon^{S}$ & - incoherent surface stress. \\
\hline$\Delta^{\perp}$ & - transverse excess strain. \\
\hline$\Sigma_{0}^{S}$ & - residual surface stress. \\
\hline$\Upsilon_{0}^{S}$ & - residual incoherent surface stress. \\
\hline$\Lambda_{0}^{\perp}$ & - residual transverse interfacial deformation. \\
\hline $\mathbb{C}^{S}$ & - interface elastic stiffness tensor. \\
\hline$\Lambda^{\perp}$ & - interface elastic compliance tensor. \\
\hline$\Upsilon_{2}^{S}$ & - interface incoherency stiffness tensor. \\
\hline$\Phi^{S}$ & - interface structural interfacial mismatch coupling tensor. \\
\hline $\mathbb{H}$ & - interfacial Poisson's effect tensor. \\
\hline $\mathbb{K}^{S}$ & - interface structural mismatch tensor. \\
\hline
\end{tabular}

\section{Complex analysis/Stroh formalism:}

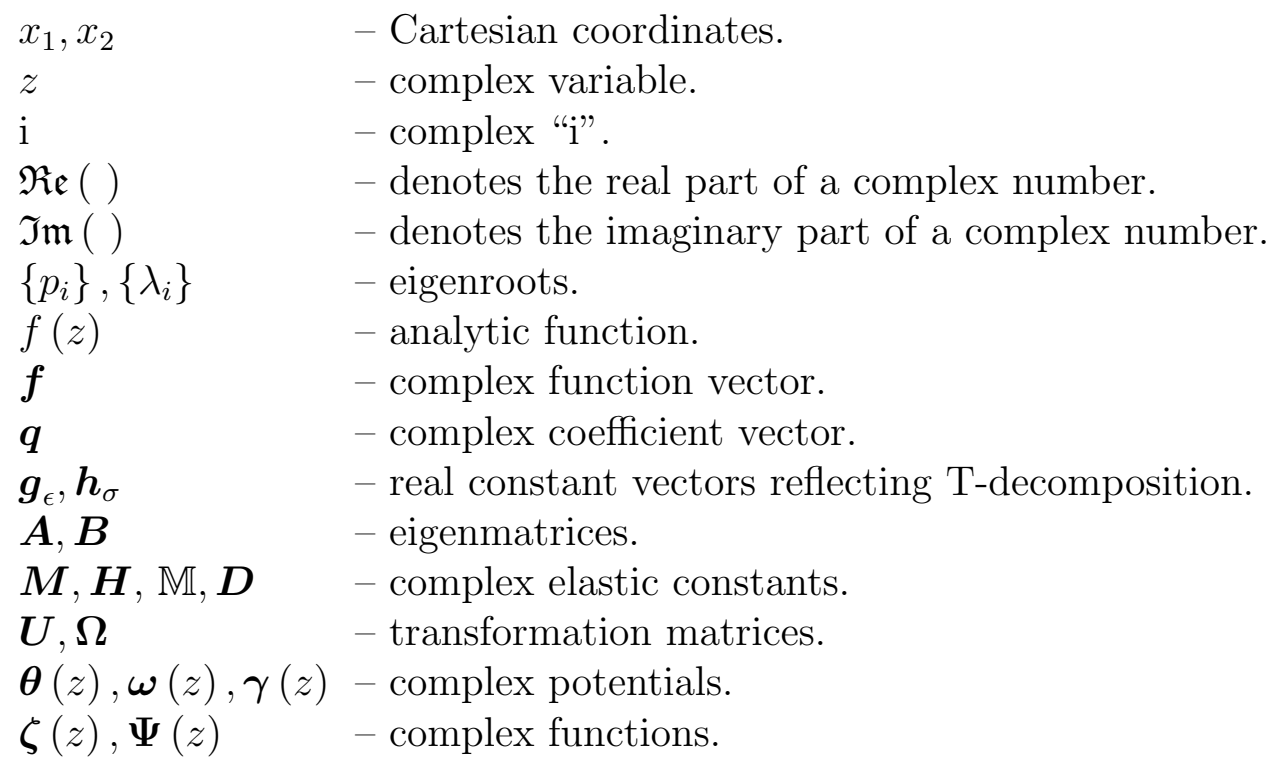




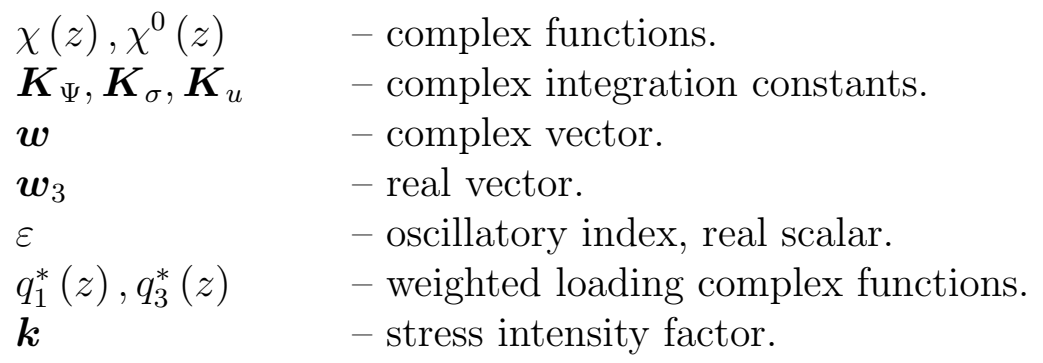

\section{Miscellaneous symbols:}

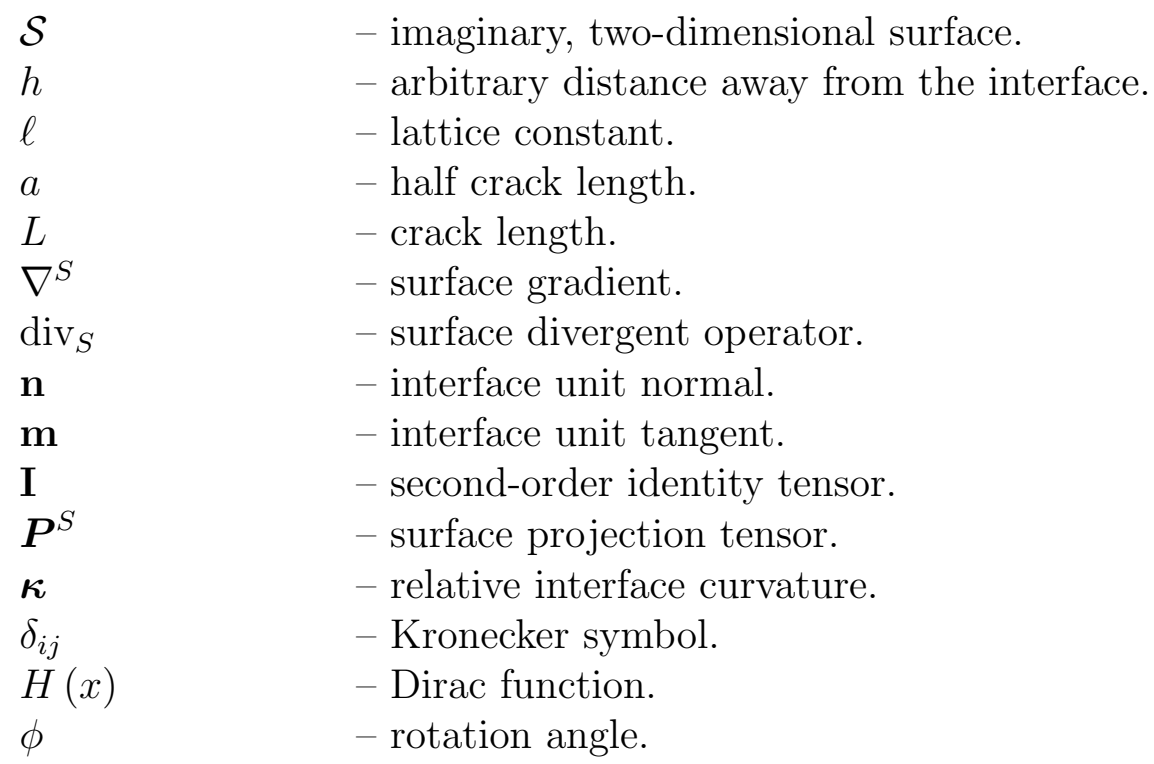

\section{Appendix B. Derivation of interfacial conditions}

Let us consider an interface $S$ separating two dissimilar linear elastic media with the normal to the interface denoted as $\mathbf{n}$. Let the area be bounded by the curve $\partial S$. The outward normal vector of $\partial S$ tangent to $S$ is denoted by $\mathbf{m}$. Within the concept of the dividing surface (Gibbs, 1928), surface contributions to the thermodynamic properties are defined as the excesses over the values that would be obtained if the bulk phases on either side of the interface retained their properties as constant up to a two-dimensional imaginary dividing surface embedded in the three dimensional continuum. In other words, the interface (to be differentiated from an interphase which has a finite thickness) is a mathematical surface with zero thickness over which the thermodynamic properties change discontinuously from one bulk phase to the other. As a consequence, in the present context of continuum mechanics, the length 
scale associated with the interphase region $h$ (considered as a region over which the material properties change gradually from the bulk property of one material to the other) can be considered infinitesimally small compared to the continuum length scale $L$ associated with finite cracks problems studied in this manuscript such that $h / L \approx 0$.

\section{Kinetic interfacial conditions}

First, let us consider the kinetic conditions at the interface. The traction vector on the top and bottom of the interface is simply given by $\boldsymbol{\sigma} \cdot \mathbf{n}$ and $-\boldsymbol{\sigma} \cdot \mathbf{n}$ respectively, while the traction along the edge of the interface is given by $\boldsymbol{\Sigma}^{S} \cdot \mathbf{m}$. The static equilibrium of the interface requires,

$$
\int_{S} \llbracket \boldsymbol{\sigma} \rrbracket \cdot \mathbf{n} d A+\int_{\partial S} \boldsymbol{\Sigma}^{S} \cdot \mathbf{m} d s=\mathbf{0} .
$$

Applying the surface divergence theorem (Gurtin and Murdoch, 1975) to the line integral defined in Eq. (B.1) yields,

$$
\int_{S}\left(\llbracket \boldsymbol{\sigma} \rrbracket \cdot \mathbf{n}+\operatorname{div}_{S}\left(\boldsymbol{\Sigma}^{S}\right)\right) d A=\mathbf{0},
$$

where $\operatorname{div}_{S}$ denotes the surface divergent operator as introduced in Gurtin and Murdoch (1975). Since Eq. (B.2) holds for any surface area $S$, we immediately obtain the following equilibrium condition on the interface,

$$
\llbracket \boldsymbol{\sigma} \rrbracket \cdot \mathbf{n}+\operatorname{div}_{S}\left(\Sigma^{S}\right)=\mathbf{0} .
$$

Equation (B.3) is the same derived as equation (6.10) derived in Gurtin and Murdoch (1975) which was derived using a variational approach. A more convenient form of Eq. (B.3) can be expressed as,

$$
\begin{aligned}
\mathbf{n} \cdot \llbracket \boldsymbol{\sigma} \rrbracket \cdot \mathbf{n} & =-\boldsymbol{\Sigma}^{S}: \boldsymbol{\kappa}, \\
\mathbf{P}^{S} \cdot \llbracket \boldsymbol{\sigma} \rrbracket \cdot \mathbf{n} & =-\nabla^{S} \boldsymbol{\Sigma}^{S},
\end{aligned}
$$

which is obtained using the following identities,

$$
\mathbf{P}^{S} \boldsymbol{\Sigma}^{S}=\boldsymbol{\Sigma}^{S}, \mathbf{n} \cdot \boldsymbol{\Sigma}^{S}=\mathbf{0}, \operatorname{div}_{S}\left(\mathbf{n} \cdot \boldsymbol{\Sigma}^{S}\right)=\mathbf{n} \cdot \operatorname{div}_{S}\left(\boldsymbol{\Sigma}^{S}\right)+\left(\nabla^{S} \mathbf{n}\right): \boldsymbol{\Sigma}^{S} .
$$

The surface projection tensor is defined as $\mathbf{P}^{S}=\mathbf{I}-\mathbf{n} \otimes \mathbf{n}$ while the surface nabla operator $\nabla^{S}$ is defined by the projection of the nabla operator $\nabla$ on the tangential plane of the interface such that,

$$
\nabla^{S}=(\mathbf{I}-\mathbf{n} \otimes \mathbf{n}) \nabla=\mathbf{P}^{S} \nabla
$$


with $\mathbf{I}$ is the second-order identity tensor. The curvature tensor of the interface $\boldsymbol{\kappa}$ is defined by,

$$
\kappa=-\nabla^{S} \mathbf{n}
$$

Kinematic interfacial conditions

Second, let us consider the kinematic interfacial conditions. Following the classical formulation of deformation tensors in curvilinear coordinates (Dingreville, 2007), the transverse strain $\boldsymbol{\epsilon}^{\perp}$ can be expressed as,

$$
\boldsymbol{\epsilon}^{\perp}=\frac{\boldsymbol{u}}{\partial x_{2}}+\mathbf{P}^{S} \cdot \nabla u_{2}+\boldsymbol{\kappa} \cdot \mathbf{P}^{S} \cdot \boldsymbol{u}
$$

The transverse interfacial excess strain $\boldsymbol{\Delta}^{\perp}$ defined in Eq. (25) is by definition expressed as,

$$
\boldsymbol{\Delta}^{\perp}=\int_{-\infty}^{0}\left(\boldsymbol{\epsilon}^{\perp}\left(x_{2}\right)-\boldsymbol{\epsilon}_{-}^{\perp}\right) d x_{2}+\int_{0}^{\infty}\left(\boldsymbol{\epsilon}^{\perp}\left(x_{2}\right)-\boldsymbol{\epsilon}_{-}^{\perp}\right) d x_{2}
$$

where $\epsilon_{ \pm}^{\perp}$ are the far field transverse strain fields in the medium + and - respectively. By making use of the above Eqs. (B.9) and (B.10), an alternative expression of the transverse interfacial excess strain is given by,

$$
\boldsymbol{\Delta}^{\perp}=\int_{-h}^{h}\left(\frac{\boldsymbol{u}}{\partial x_{2}}+\mathbf{P}^{S} \cdot \nabla u_{2}+\boldsymbol{\kappa} \cdot \mathbf{P}^{S} \cdot \boldsymbol{u}\right) d x_{2}-h\left(\boldsymbol{\epsilon}_{-}^{\perp}+\boldsymbol{\epsilon}_{+}^{\perp}\right),
$$

Carrying out the integral in Eq. (B.11) leads to,

$$
\boldsymbol{\Delta}^{\perp}=\boldsymbol{u}\left(x_{1}, h\right)-\boldsymbol{u}\left(x_{1},-h\right)+\int_{-h}^{h}\left(\mathbf{P}^{S} \cdot \nabla u_{2}+\boldsymbol{\kappa} \cdot \mathbf{P}^{S} \cdot \boldsymbol{u}\right) d x_{2}-h\left(\boldsymbol{\epsilon}_{-}^{\perp}+\boldsymbol{\epsilon}_{+}^{\perp}\right) .
$$

In the limit of the continuum scale, i.e., $h / L \approx 0$, and accounting for the uniformity of the far strain fields $\boldsymbol{\epsilon}_{+}^{\perp}$ and $\boldsymbol{\epsilon}_{-}^{\perp}$, Eq. (B.12) reduces to,

$$
\boldsymbol{\Delta}^{\perp}=\boldsymbol{u}\left(x_{1}, h\right)-\boldsymbol{u}\left(x_{1},-h\right)+O(h / L) \approx \boldsymbol{u}\left(x_{1}, h\right)-\boldsymbol{u}\left(x_{1},-h\right) \approx \llbracket \boldsymbol{u} \rrbracket,
$$

where $\llbracket \boldsymbol{u} \rrbracket$ is the jump of displacement across the interface. The displacement jump is a pure artifact of the Gibbsean description of the interface and the associated vanishing thickness of the interfacial region.

Summarizing, the interfacial conditions across the interface while accounting for the generalized interfacial elasticity formulation by Dingreville et al. (2014) consist of the following equations on the interface,

$$
\begin{aligned}
\llbracket \mathbf{u} \rrbracket & =\boldsymbol{\Delta}^{\perp}, \\
\mathbf{n} \cdot \llbracket \boldsymbol{\sigma} \rrbracket \cdot \mathbf{n} & =-\boldsymbol{\Sigma}^{S}: \boldsymbol{\kappa}, \\
\mathbf{P}^{S} \cdot \llbracket \boldsymbol{\sigma} \rrbracket \cdot \mathbf{n} & =-\nabla^{S} \boldsymbol{\Sigma}^{S}
\end{aligned}
$$

